\title{
IGF2BP1/IMP1 contributes to autophagy modulation directly via MAP1LC3B
}

Patrick A. Williams ${ }^{a}$, Louis R. Parham ${ }^{a}$, Kofi K. Acheampong ${ }^{b}$, Charles H. Danan ${ }^{a}$, Xianghui Ma ${ }^{a}$, Lauren A. Simon $^{a}$, Kaitlyn E. Naughton ${ }^{a}$, Tatiana Karakasheva ${ }^{a}$, Kelly A. Whelan ${ }^{c, d}$, Donita C. Brady ${ }^{\text {,ff }}$, Sydney M. Shaffer $^{\text {b,g }}$, Kathryn E. Hamilton ${ }^{a *}$

${ }^{a}$ Division of Gastroenterology, Hepatology, and Nutrition; Department of Pediatrics; Children's Hospital of Philadelphia; University of Pennsylvania Perelman School of Medicine, Philadelphia, PA, USA; ${ }^{b}$ Department of Pathology and Laboratory Medicine, University of Pennsylvania Perelman School of Medicine, Philadelphia, PA, USA. 'Department of Pathology \& Laboratory Medicine, Lewis Katz School of Medicine at Temple University, Philadelphia, PA, USA; ${ }^{d}$ Fels Institute for Cancer Research \& Molecular Biology, Lewis Katz School of Medicine at Temple University, Philadelphia, PA, USA. ${ }^{e}$ Department of Cancer Biology, Perelman School of Medicine, University of Pennsylvania, Philadelphia, PA, USA.

${ }^{f}$ Abramson Family Cancer Research Institute, Perelman School of Medicine, University of Pennsylvania, Philadelphia, PA, USA. ${ }^{9}$ Department of Bioengineering, University of Pennsylvania, Philadelphia, PA, USA.

${ }^{*}$ Corresponding author

Kathryn E. Hamilton, PhD

903 Abramson Research Building

Children's Hospital of Philadelphia

3615 Civic Center Blvd.

Philadelphia, PA 19104, USA

267-426-5266

hamiltonk1@chop.edu

@KHamiltonPhD

ORCID: 0000-0001-9575-2499 


\begin{abstract}
Homeostatic tissue maintenance requires coordinated regulation of metabolic processes including macroautophagy/autophagy. Autophagy dysregulation underlies numerous human diseases. Our prior work revealed that the RNA binding protein IGF2BP1/IMP1 binds transcripts encoding autophagy-related proteins. Furthermore, Imp1 deletion in gastrointestinal epithelial cells in mice was associated with enhanced autophagy flux and improved recovery from tissue injury. In the current study, we evaluated molecular mechanisms underlying IMP1 modulation of autophagy. We report increased expression of autophagy protein microtubule associated protein 1 light-chain 3B (MAP1LC3B) in cells with IMP1 deletion compared to control cells. MAP1LC3B expression was also increased in cells with mutated IMP1 phosphorylation sites. Luciferase reporter assays demonstrate increased translation of MAP1LC3B-3'UTR in cells with IMP1 deletion. Furthermore, we find that IMP1 co-localizes with MAP1LC3B transcripts at homeostasis, and co-localization is decreased in cells where IMP1 phosphorylation sites are mutated. IMP1 localization with MAP1LC3B transcripts is also decreased following cell stress. Taken together, our data support a model whereby IMP1 regulates MAP1LC3B translation which can serve as a mechanism to calibrate autophagy levels in the cell. This new mechanism may be particularly important in gastrointestinal epithelial cells, where autophagy is essential for tissue recovery following injury, or in diseases such as inflammatory bowel disease where defective autophagy is implicated.
\end{abstract}

Abbreviations IGF2 mRNA binding protein 1 (IMP1); Microtubule Associated Protein 1 Light Chain 3 Beta (MAP1LC3B) 


\section{Introduction}

Studies of the mammalian gastrointestinal tract suggest diverse roles for the autophagy pathway in both homeostasis and disease. During homeostasis, epithelial cells that line the intestine and colon utilize autophagy to maintain microbial symbiosis, secrete antimicrobial lysozyme via secretory autophagy, maintain tight junctions, and protect from DNA damage via reduction of excessive reactive oxygen species (Asano et al., 2017; Bel et al., 2017; Conway et al., 2013; Gorbunov and Kiang, 2009; Nighot et al., 2015; Sadaghian Sadabad et al., 2015). Furthermore, variants in human autophagy genes were identified as susceptibility genes in inflammatory bowel disease (Rioux et al., 2007). Mice with intestinal epithelial-specific autophagy gene deletion exhibit enhanced susceptibility to inflammatory tissue damage (Adolph et al., 2013; Asano et al., 2017; Cadwell et al., 2008; Conway et al., 2013; Lassen et al., 2014; Trentesaux et al., 2020). Conversely, factors that increase autophagy, such as nutrient deprivation, enhance intestinal/colonic tissue regeneration (Rangan et al., 2019; Yousefi et al., 2018). These data together underscore diverse and important roles for autophagy in gastrointestinal epithelial cells, however, regulation of autophagy in the gut is incompletely understood.

Individual components of the autophagy pathway are regulated at multiple levels (Copetti et al., 2009; Feng et al., 2015; Lipinski et al., 2010; Yee et al., 2009). Emerging studies demonstrate post-transcriptional regulation of autophagy via long non-coding RNAs, microRNAs, and RNA binding proteins (RBPs) (Jing et al., 2015). Cytoplasmic RBPs regulate mRNA stability, localization, and translation by governing interactions with the translation machinery, cytoskeletal network, or other trans-acting factors that ultimately direct temporal and spatial protein translation. As such, RBPs contribute to an essential mechanism for cells to preferentially translate mRNAs in response to external stimuli or stress. Defining how RBPs contribute to autophagy regulation in specific cell types, such as gastrointestinal epithelial cells, could provide a basis for new therapies for gastrointestinal diseases.

Insulin-like growth factor 2 mRNA-binding protein 1 (IGF2BP1/IMP1) is an RBP expressed broadly during development and in human tumors, but its expression in adult tissues is low or absent(Bell et al., 2013). Our recent studies demonstrate upregulation of IMP1 in adult tissue including the intestinal/colonic stem cell niche during tissue damage in mice and in patients with inflammatory bowel disease (Chatterji et al., 2018; Chatterji et al., 2019). Studies in cell lines suggest a primary role for IMP1 to regulate cell proliferation, however, in vivo analyses revealed little effect on cell proliferation in mice with intestinal epithelial cell-specific deletion of $\operatorname{Imp} 1\left(\operatorname{Imp} 1^{\triangle I E C}\right)$ at homeostasis. Instead, the intestinal epithelium of $I m p 1^{4 I E C}$ mice exhibits increased autophagy flux associated with a decrease in intestinal tissue damage compared to control mice (Chatterji et al., 2018; Chatterji et al., 2019). IMP1 modulation of autophagy is consistent with in vitro studies implicating a role for IMP1 during cell stress and studies showing that cytoplasmic IMP1-granules contain transcripts encoding secretory pathway genes that may be related to the autophagy pathway(Jonson et al., 2007; Stohr et al., 2006; Zeng et al., 2020). While our prior data support the notion that IMP1 can act as a modulator of autophagy in gastrointestinal epithelial cells, a direct mechanism remains unclear.

Our goal in the present study was to evaluate direct regulation of autophagy transcripts by IMP1. We evaluated IMP1 regulation of autophagy at the RNA and protein levels and via direct translational regulation using dual-luciferase reporter assays. We examined the relative contribution of phosphorylated IMP1 ( $\mathrm{p}-\mathrm{IMP} 1$ ), known to regulate transcript binding and translation in other contexts (Dai et al., 2013; Huttelmaier et al., 2005; Lambrianidou et al., 2021), to autophagy protein expression and IMP1 cytoplasmic localization. Finally, we evaluated IMP1 co-localization with autophagy transcripts at homeostasis and in response to arsenite-induced cell stress. Herein, we report new data to support a previously unknown mechanism of autophagy pathway modulation via post-transcriptional regulation of MAP1LC3B by IMP1. 


\section{Results}

\section{IMP1 knockout cells exhibit increased MAP1LC3B protein expression}

Prior work from our group demonstrated that IMP1 deletion in vivo results in increased autophagy flux. In addition, we found via ribonucleoprotein immunoprecipitation (RIP)-qPCR that IMP1 can bind directly to autophagy transcripts ATG5, ATG3, and MAP1LC3B (Chatterji et al., 2019). The downstream effects of IMP1 binding to specific autophagy transcripts in not known. We evaluated the consequence of IMP1 deletion on MAP1LC3B protein expression by examining IMP1 wild type (IMP1-WT) and IMP1 knockout (IMP1-KO) SW480 colon cancer cells. We used two different protein concentrations for immunoblotting to allow for quantification, with the lower concentration giving for better band resolution. Using an antibody specific to MAP1LC3B, we observed a significant increase the phosphatidylethanolamine(PE)-conjugated form of LC3 (LC3-II) in IMP1-KO compared to IMP1-WT cells ( $3.2 \pm 0.32$ vs $1.0 \pm 0.12$, p <0.0001), an effect that was increased with torin (3 hours), an mTOR inhibitor that induces autophagy (Thoreen et al., 2009). We also observed an increase in LC3-II in cells treated with V-ATPase inhibitor bafilomycin $\mathrm{A}_{1}$, which inhibits autophagosome-lysosome fusion (Klionsky et al., 2008; Yamamoto et al., 1998), in IMP1-KO compared to IMP1-WT cells $(4.9 \pm 0.24$ vs $2.8 \pm 0.23, p<0.0001)$ (Figure 1A and 1B).

An increase in LC3-II in untreated cells can be interpreted as either increased autophagosomes or blocked autophagy, however, the further increase in LC3-II in IMP1-KO cells with bafilomycin $\mathrm{A}_{1}$ treatment suggests increased autophagosomes/autophagy flux (Klionsky et al., 2021; Mauvezin and Neufeld, 2015; Mizushima and Yoshimori, 2007; Yoshii and Mizushima, 2017). We used a second antibody with reactivity to both MAP1LC3A and MAP1LC3B to evaluate LC3-II with saturating doses of bafilomycin $\mathrm{A}_{1}$ treatment for 24 hours. In this case, there was little difference between cell lines (Figure 1C \& 1D) suggesting that the MAP1LC3A isoform is not affected by IMP1 deletion and use of an antibody targeting both MAP1LC3A and MAP1LC3B instead of MAP1LC3B-only leads to a diminution in phenotype with IMP1-KO. Despite the muted phenotype by immunoblot with MAP1LC3A/B antibody detection, immunostaining using the MAP1LC3A/B antibody revealed increased puncta in IMP1-KO versus IMP1-WT cells (Figure 1F), supporting an overall effect of IMP1-KO to increase MAP1LC3B. Immunoblot analysis of other IMP1 targets ATG5 and ATG3 revealed no change between IMP1-WT and IMP1-KO cells (Supplemental Figure 1A). Taken together, these data suggest that IMP1-KO has a specific effect on MAP1LC3B compared to MAP1LCA or other its other putative RNA-binding targets, and that this effect may contribute to MAP1LC3 protein localization into puncta.

We next evaluated cells treated with exposure to torin (24 hours). We observed an increase in LC3II between IMP1-WT and IMP1-KO cells at baseline and with torin treatment (Supplemental Figure 1A \& B), however, cargo-associated protein p62 appeared higher in untreated IMP1-KO cells compared to IMP1WT cells, indicated that at this time point, IMP1-KO cells may exhibit stalled autophagy flux. Taken together, results in Figure 1 indicate that at longer timepoints post-plating, autophagy flux could ultimately be saturated, rather than increased, in IMP1-KO compared to IMP1-WT cells. This is intriguing, since our prior studies in mouse organoids from Imp1 $1^{\Delta \mathrm{EC}}$ mice demonstrate enhanced autophagy flux (Chatterji et al., 2019). As such, it is possible that in longer cell culture intervals, a compensatory mechanism may be activated in IMP1-KO cells. Finally, evaluation of MAP1LC3B mRNA levels with real-time RT-PCR revealed no difference in MAP1LC3B expression in IMP1-KO compared to IMP1-WT cells (Supplemental Figure 1C), indicating that IMP1-KO is associated with increased MAP1LC3B primarily at the protein level. Taken together, we observe increased LC3-II levels with IMP1-KO via specific effects on MAP1LC3B, and not MAP1LC3A, ATG3, or ATG5, protein levels. Analysis of shorter timepoints post-plating and post-treatment reveal that upregulation may result in enhanced autophagy flux initially, whereas longer timepoints postplating or post-treatment is associated with stalled autophagy flux based on accumulation of cargoassociated protein p62 (Klionsky et al., 2021).

\section{IMP1 phosphorylation contributes to its modulation of MAP1LC3B protein expression}

IMP1 contains a mTORC2-dependent phosphorylation site at serine 181 (S181) and a Src kinasedependent site at tyrosine 398 (Y396) (Figure 2A), which can affect translation and/or localization of IMP1 targets (Dai et al., 2013; Huttelmaier et al., 2005; Lambrianidou et al., 2021). We therefore evaluated the relative contribution of IMP1 phosphorylation (p-IMP1) to its modulation of MAP1LC3B. We generated IMP1-KO HEK293 cell lines and introduced the following constructs: control-GFP (IMP1-KO), IMP1-WTGFP, and phosphorylation mutants: IMP1-S181A-GFP, IMP1-Y396F-GFP, or double-mutant IMP1S181A/Y396F-GFP. We observed increased MAP1LC3B protein expression in control-GFP (IMP1-KO, 
$3.282 \pm 0.91), I M P 1-Y 396 F-G F P(1.45 \pm 0.31)$ and IMP1-S181A/Y396F-GFP $(3.95 \pm 1.38)$ mutant cells compared to IMP1-WT-GFP $(1.0 \pm 0.31)$, or IMP1-S181A-GFP $(0.83 \pm 0.45)$, with statistical significance in IMP1-Y396F-GFP versus IMP1-WT-GFP cells (Figure 2B\&C). Taken together, these data suggest that IMP1-Y396 contributes to IMP1 modulation of MAP1LC3B protein expression.

We next used "Autophagy Assay, Red", a fluorescent probe that is activated when inserted into lipid membranes of autophagosomes and autolysosomes (Supplemental Figure 2A) (Czarnomysy et al., 2020), to determine the functional relevance of p-IMP1 for autophagy flux. We treated control-GFP (IMP1KO), IMP1-WT-GFP, IMP1-S181A-GFP, IMP1-Y396F-GFP, or IMP1-S181A/Y396F-GFP cells with 100 $\mu \mathrm{M}$ chloroquine (CQ) for 3 hours to inhibit lysosomal degradation, where accumulation of autophagic vesicles serves as an indicator for levels of autophagy flux (Mauthe et al., 2018). We observed a greater increase in autophagic vesicle accumulation in control-GFP (IMP1-KO), IMP1-Y396F-GFP, and IMP1-S181A/Y396FGFP cells compared to IMP1-WT-GFP or IMP1-S181A-GFP cells, with statistically significant increases in GFP (IMP1-KO), IMP1-Y396F-GFP cells. These data together support the premise that IMP1-Y396, but not -S181 contributes to IMP1's regulation of MAP1LC3B, which can ultimately contribute to modulation of autophagy flux.

We next evaluated how p-IMP1 mutation affects its cellular localization, since prior studies suggest that IMP1 phosphorylation modulates IMP1 regulation of MYC (Lambrianidou et al., 2021). Imaging of GFP expression revealed primarily cytoplasmic staining in IMP1-WT-GFP, IMP1-Y396F-GFP, and IMP1S181A/Y396F-GFP cells. IMP1-S181A-GFP cells exhibited a decrease in cytoplasmic staining with concomitant GFP expression in the nucleus (Figure 3A). Using cell fractionation followed by western blotting, we confirmed reduced cytoplasmic expression in IMP1-S181A-GFP cells compared to IMP1-WTGFP cells (Supplemental Figure 3A-B). While IMP1 localization differences in IMP1-S181A-GFP cells is intriguing, we ultimately utilized IMP1-WT-GFP and IMP1-S181A/Y396F-GFP cells in subsequent mechanistic studies since IMP1-S181A did not affect MAP1LC3B expression.

\section{IMP1 regulates MAP1LC3B in part via direct modulation of MAP1LC3B-3'UTR translation}

Our previous ribosome-profiling data suggested that autophagy pathway translation is enhanced in IMP1-KO cells (Chatterji et al., 2019). Furthermore, analysis of published enhanced UV crosslinking and immunoprecipitation (eCLIP) studies showed enrichment of IMP1 binding sites in the MAP1LC3B-3'UTR (Chatterji et al., 2019; Conway et al., 2016). We therefore evaluated whether IMP1 deletion was associated with increased translation of MAP1LC3B through the 3'UTR using a dual-luciferase reporter system (Figure 3B). We found that IMP1-KO (1.37 \pm 0.1$)$ and IMP1-S181A/Y396F-GFP (1.39 \pm 0.24$)$ cells transfected with MAP1LC3B-3'UTR exhibited increased luciferase reporter activity compared to IMP1-WT cells (1.09 \pm 0.16 ; Figure 3C). Taken together, these data suggest that IMP1 may modulate MAP1LC3B in part via direct modulation of MAP1LC3B-3'UTR.

\section{IMP1 phosphorylation contributes to dynamic localization of IMP1 in response to cell stress}

Prior studies indicate that IMP1 can localize to mRNA-containing granules (mRNP granules) including p-bodies (PBs) and stress granules (SGs), which can be sites of translational quiescence and/or mRNA degradation (Bley et al., 2015; Hubstenberger et al., 2017). Under homeostatic conditions, IMP1WT-GFP signal is mostly diffuse, however, we observed a small number of granules positively stained with the PB marker EDC4 that co-localized with IMP1-WT-GFP puncta (Figure 4A, vehicle, white arrows). Treatment with sodium arsenite (which elicits oxidative stress and can induce autophagy) (Bolt et al., 2010), results in increased GFP abundance in larger, more punctate granules that exhibit reduced overlap with EDC4+ granules denoted by non-overlapping red and green puncta (Figure 4A, arsenite-treated). GFP expression and localization appeared similar in IMP1-S181A/Y396F-GFP cells in both vehicle and sodium arsenite conditions and overlap with EDC4+ puncta were similarly decreased with sodium arsenite treatment (Figure 4B). There were fewer apparent EDC4 puncta in IMP1-S181A/Y396F-GFP cells in both vehicle and sodium arsenite conditions compared to IMP1-WT-GFP cells, indicating that while localization of IMP1 in PBs does not appear to be altered by mutant p-IMP1, there may be a general effect of mutant $p$ IMP1 on PB dynamics.

IMP1 has been described previously to localize in SGs and to be increased in SGs following cell stress (Bley et al., 2015). We therefore evaluated co-staining of IMP1 with SG marker PABP. As anticipated, a majority of IMP1 appeared to co-localize with PABP-marked granules following sodium arsenite treatment in IMP1-WT-GFP cells (Figure 4C, white arrows in insets in arsenite-treated cells). We observed reduced overall granule induction in IMP1-S181A/Y396F-GFP cells (Figure 4D), though the 
granules that did form exhibited overlap between GFP and PABP (white arrows in insets with sodium arsenite treatment). Finally, since we observed an overall reduction in granule formation in response to sodium arsenite in mutant p-IMP1 cells, we performed time-lapse imaging of GFP in sodium arsenitetreated cells over 1.5 hours in IMP1-WT-GFP, IMP1-S181A-GFP, and IMP1-Y396F-GFP expressing cells. IMP1-WT-GFP cells exhibited robust cytoplasmic granule formation with sodium arsenite treatment (Movie S1), whereas granule formation appeared modestly reduced, but still intact, in IMP1-S181A-GFP, and IMP1-Y396F-GFP expressing cells (Movie S2 and S3). Collectively, these data support a role for p-IMP1 in contributing to cytoplasmic granule formation in response to stress, but its localization in specific granules warrants further investigation.

\section{Cytoplasmic IMP1 co-localizes with MAP1LC3B transcript}

Due to the complexity of p-IMP1's contribution to its localization in specific granules, we instead decided to evaluate whether IMP1 co-localizes with MAP1LC3B transcripts using single molecule mRNA FISH (smFISH) combined with immunofluorescent imaging of GFP. We observed co-localization of IMP1GFP with MAP1LC3B transcripts (Figure 5A, white arrows), but not ATG5 transcripts (Figure 5B). IMP1S181A/Y396F-GFP cells exhibited reduced GFP co-localization with MAP1LC3B transcripts (Figure 5C). Arsenite treatment was associated with re-distribution of MAP1LC3B mRNA away from IMP1 granules in IMP1-WT-GFP cells (Figure 5A), while IMP1-S181A/Y396F-GFP cells exhibited less overall cytoplasmic IMP1 (Figure 5C). IMP1 did not co-localize with ATG5 mRNA in arsenite-treated cells (Figure 5D). We quantified co-localization between IMP1-GFP and MAP1LC3B fluorescence using rank weight coefficient and found significantly more overlap between MAP1LC3B and IMP1-WT-GFP compared to IMP1-

S181A/Y396F-GFP (Figure 5E). Finally, we found that MAP1LC3B mRNA was distributed closer to the cell nucleus in IMP1-WT-GFP cells compared to IMP1-S181A/Y396F-GFP cells or IMP1-WT-GFP cells treated with arsenite (Figure 5F), supporting the notion that mutation of $p$-IMP1 mimics arsenite-induced cell stress. Taken together, these data suggest that at homeostasis, IMP1 contributes to MAP1LC3B transcript localization closer to the nucleus, whereas arsenite-induced stress appears to redistribute MAP1LC3B transcripts away from the nucleus, presumably when it is no longer sequestered by IMP1.

\section{Discussion}

Studies presented herein define new molecular mechanisms underlying our prior observation that autophagy flux is upregulated in intestinal epithelial cells in mice with IMP1 deletion. Prior results from our lab suggested direct binding of IMP1 to multiple autophagy transcripts (Chatterji et al., 2019). In the present manuscript, we evaluated autophagy genes at the protein and RNA level in cells with IMP1 deletion and found a robust increase in microtubule associated protein 1 light-chain 3B (MAP1LC3B) protein compared to other autophagy targets. Real-time RT-PCR results, where MAP1LC3B expression was not changed with IMP1 deletion, suggest that IMP1 modulates MAP1LC3B almost exclusively at the protein level. Furthermore, mutation of the IMP1 Y-396 phosphorylation site, either alone or together with mutation in the IMP1 S-181 phosphorylation site, recapitulated the increase in MAP1LC3B we observed with IMP1 deletion. We next evaluated the effect of IMP1 deletion on MAP1LC3B translation using a dual luciferase reporter assay. We focused on the 3'UTR of MAP1LC3B based on our analysis of eCLIP data published by others (Conway et al., 2016), which showed a propensity of IMP1 binding at MAP1LC3B-3'UTR versus the coding sequence or 5'UTR (Chatterji et al., 2019). Our data demonstrate that cells with IMP1 deletion exhibit higher translational activity for MAP1LC3B-3'UTR than in cells with wildtype IMP1 levels. This could be due to, at least in part, to IMP1 localizing out of translationally quiescent processing bodies and away from MAP1LC3B transcripts in response to stress, as demonstrated by co-staining with IMP1 with processing body marker EDC4. We used single molecule mRNA FISH to evaluate IMP1 protein localization with MAP1LCB3 transcripts. We report that IMP1 co-localizes with MAP1LCB3B transcripts at homeostasis, and that both stress induction via sodium arsenite or mutation of p-IMP1 sites is associated with decreased co-localization. Furthermore, MAP1LC3B transcripts exhibit different localization in IMP1WT versus p-IMP1 mutant or arsenite-treated cells, localizing closer to the nucleus in IMP1-WT cells. Taken together, our results support the notion that IMP1 modulates autophagy at homeostasis via direct regulation of MAP1LC3B translation and localization in a manner dependent, at least in part, upon p-IMP1 (Figure 6).

MAP1LC3 (also known as Atg8 in yeast) is a component of the autophagy cascade that, upon conjugation with phosphatidylethanolamine (PE), is essential for autophagosome formation and expansion (Ichimura et al., 2000; Nakatogawa et al., 2007). There are three human isoforms of MAP1LC3 (LC3A, 
LC3B, LC3C) with differing expression patterns in tissue, all of which are associated with autophagic membranes in the cell (He et al., 2003). Our prior published study showed specific binding of IMP1 to the MAP1LC3B isoform (Chatterji et al., 2019). While mechanisms regulating expression and localization of MAP1LC3B protein are reported (Drake et al., 2010; Huang et al., 2015), post-transcriptional regulation of MAP1LC3B is only beginning to be elucidated. For example, knockdown of BCL2-associated athanogene 3 (BAG3), a co-chaperone of the HSP70 system, was associated with decreased MAP1LC3B protein, but not mRNA, although the direct mechanism is not yet known (Rodriguez et al., 2016). Others reported regulation of MAP1LC3B transcripts by the microRNA mir204 (Mikhaylova et al., 2012). We find that IMP1 deletion is associated with increased MAP1LC3B expression primarily at the protein level. Importantly, the increase in LC3-II observed with IMP1 deletion does not appear to be attributed to impaired autophagy, as treatment with autophagy inhibitor bafilomycin A was associated with accumulation of LC3 and p62 in both control and IMP1-KO cells. As such, IMP1 appears to regulate the total amount of MAP1LC3B protein that is expressed, which could allow for more autophagy flux at homeostasis.

IMP1 is regulated in part via phosphorylation by either mTORC2 at S-181 or Src at Y-396 (Dai et al., 2013; Huttelmaier et al., 2005). Others reported recently diffuse cytoplasmic IMP1 in IMP1-S181A cells, and increased granule formation in IMP1-Y396F cells (Lambrianidou et al., 2021). In our hands, we confirm that IMP1-S181 contributes to diffuse cytoplasmic IMP1, however, we found additionally that cytoplasmic IMP1 decreased, and nuclear IMP1 expression increased, in IMP1-S181 mutant cells. More importantly, we did not observe a difference in MAP1LC3B protein expression or autophagy levels in IMP1-S181 mutant cells. Rather, mutation of IMP1-Y396 alone, or together with mutant IMP1-S181, was associated with increased MAP1LC3B expression and increased autophagy, similar to what is observed in IMP1-KO cells. Differences in IMP1 localization patterns between the present study and Lambrianidou et al. could be due to expression of phospho-mutants on an IMP1-KO versus IMP1-WT background, respectively. Taken together, we demonstrate a mechanistic link between IMP1 phosphorylation by Src and its regulation of MAP1LC3B protein expression.

Previous reports demonstrate IMP1 expression in processing bodies (PBs), which are typically translationally inactive (Hubstenberger et al., 2017), or in stress granules (SGs), which are dense RNAprotein aggregates that form during cell stress (Guzikowski et al., 2019; Niewidok et al., 2018; Stohr et al., 2006). IMP1-containing granules may also represent an entity distinct from PBs, SGs, or other granules (Jonson et al., 2007). In addition, several studies suggest IMP1 contributes to "post-transcriptional regulons" via its presence in granules containing multiple different transcripts, whereas another recent study suggested IMP1 localization in granules with mRNA singletons (Mateu-Regue et al., 2019). While its localization remains complex and requires further investigation, increased IMP1 expression during cell stress has been shown in multiple studies, including our own, (Bley et al., 2015; Wachter et al., 2013; Zeng et al., 2020). As such, IMP1 may contribute to "triaging" transcripts for translation as a mechanism to respond to cell stress. We find that IMP1 partially overlaps with PB marker EDC4 at homeostasis, but IMP1-expressing puncta are redistributed away from EDC4 granules following stress induction with sodium arsenite. We find fewer EDC4+ puncta in p-IMP1 mutant cells, however mutant p-IMP1 does still colocalizes with EDC4. We observed the same effect for IMP1 with SG marker PABP in arsenite-treated cellsan overall decrease in granules with mutant $\mathrm{p}$-IMP1, but intact co-localization. Time-lapse imaging of IMP1WT-GFP or p-IMP1 mutant cells confirm only partial impairment of IMP1 accumulation into puncta following sodium arsenite treatment. Prior studies suggest that IMP1 is dispensable for SG assembly (Bley et al., 2015). Since it is known that IMP1 localizes to multiple cytoplasmic granule subtypes, mutation of p-IMP1 could affect dynamic movement of IMP1 to multiple different granules in response to stress, resulting in a net decrease of these granules rather than a specific decrease in SGs (Bley et al., 2015). Taken together, we observed dynamic movement of cytoplasmic IMP1 in response to stress that appears to depend, at least in part, upon p-IMP1.

Finally, our finding that IMP1-WT-GFP colocalizes with MAP1LC3B mRNA and not ATG5 mRNA supports the notion that IMP1 specifically associates with MAP1LC3B transcripts. Sodium arsenite-induced stress decreased IMP1 localization with MAP1LC3B mRNA, suggesting that during stress, IMP1 no longer complexes with MAP1LC3B mRNA. We find reduced IMP1-MAP1LC3B co-localization following stress or in p-IMP1 mutant cells. This is intriguing, as recent studies suggest a functional role for localized translation of specific transcripts in polarized epithelium, such as intestinal epithelial cells (Moor et al., 2017). We find that there is more MAP1LC3B transcript near the nucleus in IMP1-WT cells compared to $p$-IMP1 mutant cells or wildtype cells treated with sodium arsenite. Based on these findings in adherent cells, follow-up studies will 
evaluate whether IMP1 contributes to specific localization of MAP1LC3B mRNA at apical versus basolateral compartments at homeostasis and in response to cell stress.

In summary, we report a new mechanism for post-transcriptional MAP1LC3B regulation. IMP1 sequestration of MAP1LC3B in cytoplasmic granules may poise homeostatic cells to respond to stress by protecting MAP1LC3B mRNA, and upon stress, releasing MAP1LC3B mRNA for translation. Our prior work demonstrated that IMP1 is expressed in a small, yet important, subset of cells in the intestine- namely stem cells and other cells in the intestinal crypt base. Both intestinal stem cells and Paneth cells may utilize the autophagy pathway for different functions (Asano et al., 2017; Bel et al., 2017; Trentesaux et al., 2020). In addition, recent studies demonstrate that autophagy is upregulated with IMP1 deletion in macrophages (Liu et al., 2022). As such, IMP1 modulation of MAP1LC3B could exhibit variable functional consequences depending on cell type expression, cell function, and upstream signaling. Studies evaluating the interplay between autophagy and post-transcriptional mechanisms continue to emerge (reviewed in (Frankel et al., 2017) and (Abildgaard et al., 2020)). Several pathologies, including neurodegeneration and inflammatory bowel disease (IBD) are associated with abnormal or defective autophagy. Future studies in human tissues and/or organoids are required to place the IMP1-MAP1LC3B regulatory axis into the context of human diseases. Understanding post-transcriptional mechanisms of autophagy regulation in tissues such as the gastrointestinal tract will provide a basis for understanding human disease pathogenesis, and ultimately, new therapeutic strategies for these diseases.

\section{Acknowledgments}

The authors thank Dr. Joseph Baur for helpful discussions and feedback. The authors thank Drs. Sarah Andres and Todd Strochlic for providing plasmid backbones used in the present studies.

\section{Funding}

NIH R01DK124369 (KEH); NIH R21ES031533 (KEH); Institutional Development Funds from Children's Hospital of Philadelphia Research Institute (KEH); Penn Center for Molecular Studies in Digestive and Liver Diseases NIH P30DK050306 (KEH); NIH R01DK12115 (KAW); NIH DP5OD028144 (SMS).

\section{Author Contributions}

Study concept and design: Williams, Hamilton. Acquisition of data: Williams, Parham, Acheampong, Ma, Simon, Naughton, Shaffer. Analysis and interpretation of data: Williams, Parham, Acheampong, Ma, Simon, Danan, Karakasheva, Whelan, Shaffer, Hamilton. Drafting of the manuscript: Williams, Hamilton. Analysis of the manuscript for intellectual and conceptual content: Williams, Whelan, Brady, Hamilton. Study Supervision: Hamilton.

\section{Declaration of Interests}

The authors declare no conflicts.

\section{Methods \\ Generation of HEK293 and SW480 IMP1-KO cell lines}

Indicated cells were seeded into a 6 -well plate and grown to $60 \%$ confluency under standard conditions: growth medium- DMEM with $4.5 \mathrm{~g} / \mathrm{L}$ glucose and L-glutamine with $10 \mathrm{mM}$ sodium pyruvate (Corning \#10017-CV), 10\% FBS (Peak Serum PS-FB1), and Penn/Strep (Gibco \#15140122), and in 5\% $\mathrm{CO}_{2}$. IMP1 deletion was performed using the IGF2BP1 CRISPR/Cas9 knockout plasmid (Santa Cruz Cat\# sc-401703) and IGF2BP1 HDR Plasmid (Santa Cruz Cat\# sc-401703-HDR) protocol as described by the manufacturer. Cells were selected using $2 \mathrm{ug} / \mathrm{mL}$ of puromycin for 2 passages and knockout was confirmed using western blotting.

\section{Generation of cell lysates and western blot}

Cells were lysed with lysis buffer (Cell Lysis reagent (Cell Signaling \#9803S), Halt Protease Inhibitor Cocktail (Life technologies \#78430), 1 mM Sodium Orthovanadate, 10 uM Sodium Fluoride) on ice and spun at $9391 \mathrm{rcf}$ at $4^{\circ} \mathrm{C}$ for 10 minutes. Running buffer (final concentration $62.5 \mathrm{mM}$ Tris-HCl pH 6.8, 2.5\% SDS, $0.002 \%$ Bromophenol Blue, 5\% B-mercaptoethanol, and 10\% glycerol) was added to supernatant and boiled for 6 minutes and loaded onto an SDS-PAGE gel with a percentage of $10 \%, 12.5 \%$, or $15 \%$ acrylamide. Samples were run and transferred onto PVDF membrane using the Bio-rad Trans-blot Turbo system and then blocked in either $5 \%$ Milk or BSA in TBS-T (20.7 mM Tris Base, $150.7 \mathrm{NaCl}, 0.1 \%$ Tween- 
20, $\mathrm{pH}$ 7.6) for 1 hour at room temperature. Primary antibodies were diluted in 5\% Milk or BSA in TBS-T and incubated with gentle rocking at $4^{\circ} \mathrm{C}$ for 24 hours. Blots were washed with TBS-T and then incubated in appropriate secondary antibody for 1 hour at room temperature with rocking. Following washing, blots were incubated with luminol reagent (Santa Cruz Biotechnology sc-2048) per manufacturer protocol and exposed to autoradiography film. All antibodies are listed in Table 1.

\section{Real- time RT-PCR}

Total RNA was isolated from cells using the Quick-RNA Mini-Prep Kit (Zymo Research R1054) and cDNA was made using the High-Capacity cDNA reverse Transcription Kit (Applied Biosystems \#4368813). Realtime PCR assays were performed on cDNA using the TAQMAN Fast Gene Expression Universal PCR Master Mix 2X (Applied Biosystems \#4352042) per the manufacturer protocol in conjunction with the Applied Biosystems Step-One Plus. Gene expression was calculated using $R=2^{(-\mathrm{DDCt})}$ method. All $\mathrm{qPCR}$ assays are listed in Table 2.

\section{Plasmid construct generation}

IMP1-GFP was cloned by amplifying PB-FLAG-IMP1 - short 3'UTR (obtained from Dr. Sarah Andres, OHSU) using primers (F'-CCTGGTACCATGAACAAGCTTTACATC and

R'ACGCATGAATTCCTTCCTCCGTGCCTGGGCCTG) and was purified from $1 \%$ agarose gel. Fragment was digested using EcoRI and Kpnl and ligated into pcDNA3-eGFP (Addgene) and sequenced. IMP1S181A-GFP and IMP1-Y396-GFP were generated by site directed mutagenesis of IMP1-GFP by Genewiz LLC, and IMP1-S181A/Y396F-GFP was generated by site directed mutagenesis of IMP1-S181A-GFP by Genewiz LLC. 3'UTR luciferase constructs were generated by insertion through restriction digestion of a synthetic peptide corresponding to appropriate gene sequence into psiCHECK-2 (Promega C8021) at the Xhol and Notl sites by Genewiz LLC.

\section{Generation of HEK293- IMP1-GFP mutant cells}

HEK293-IMP1-KO cells described above were used to seed 6-well plates to $60 \%$ confluency. Cells were then transfected with pcDNA-GFP, pcDNA-IMP1-GFP, pcDNA-IMP1-S181A-GFP, pcDNA-IMP1-Y396FGFP, or pcDNA-IMP1-S181A/Y396F-GFP plasmids using lipofectamine 3000 and 2.5 ug of DNA/well for 24 hours in DMEM+10\% FBS. Following 24 hours transfection, media was replaced with standard media (DMEM, 10\% FBS, Penn/Strep) for 24 hours. Selection with $500 \mathrm{ug} / \mathrm{mL}$ G418 occurred for $4-5$ days to enrich for a stably expressing population. After selection, cells were sorted for highest $5 \%$ GFP expression using Zombie Aqua (BioLegend 423101) and the MoFlo Astrios (Beckman Coulter).

\section{Immunofluorescence imaging}

Cells were grown on sterilized glass coverslips in 6-well plates under standard conditions until $70 \%$ confluent, washed with 1X PBS and fixed with 4\% paraformaldehyde (thermoscientific J19943-K2) for 30 minutes at room temperature. Cells were washed again with PBS and then permeabilized in $0.1 \%$ triton X100 for 5 minutes and then $0.5 \%$ triton $X-100$ for 20 minutes before washing with PBS. Cells blocked in $3 \%$ BSA for 1 hour, then incubated in primary at $4^{\circ} \mathrm{C}$ for 24 hours followed by washing and incubation with secondary antibody for 30 minutes. Cells stained with DAPI and mounted to coverslips using VECTASHIELD Vibrance Antifade Mounting Medium (Vector Laboratories H-1700-2) and sealed with nail polish (Wet'n'Wild) and imaged using a Keyence BZ-X700 microscope. Images were processed using BZ-X Analyzer (Keyence).

\section{Cellular fractionation assay}

Cells were grown in 6-well plates until reaching $80 \%$ confluency. Cells were trypsinized, pelleted and washed with PBS. Cell pellets were used for Subcellular Protein Fractionation Kit for Cultured Cells (Thermo Scientific Cat\# 78840) per the manufacturer instructions and lysates were run on a denaturing gel.

\section{Autophagy Assay Kit (Red) for measuring autophagic flux}

To evaluate autophagic flux, HEK293T cells were treated either with DMSO (control) or chloroquine (100uM) for 3 hours before being collected and stained with the Autophagy Probe, Red for subsequent flow cytometric analysis. Autophagy Probe, Red kit (Abcam, ab270790) was performed according to manufacturer's instructions. One vial of the provided Autophagy Assay, Red powder was reconstituted in $100 u$ u of DMSO to form a $250 X$ stock solution. Immediately before staining, a $50 X$ solution of the probe was 
prepared in PBS and 10ul of the solution was added to 490uL of each sample cell suspension. The samples were shielded from light and incubated for 30 minutes at $37^{\circ} \mathrm{C}$. Following staining, cells were washed and resuspended in 500ul of 1X PBS with 4\% FBS and were analysed via flow cytometry. Dead cells were excluded from the analysis with the use of DAPI staining. Samples with less than $50 \%$ viability were excluded from analyses. Flow cytometry was performed on the LSRFortessa (BD Bioscience) and analysed with FlowJo software. Data were analyzed by comparing the median fluorescence intensity (MFI) of untreated (control) and chloroquine (CQ) treated samples. The fold change in MFI between each treatment group was calculated and was compared using an ordinary one-way ANOVA with Dunnett's multiple comparison test.

\section{Luciferase translation assay}

HEK293 IMP1-WT or KO were grown in 12-well plates to $60 \%$ confluency followed by transfection with previously described siCHECK2 constructs using lipofectamine 3000 reagent (Thermofisher Cat\#L300008) according to manufacturer protocol at $0.5 \mathrm{ug}$ of DNA per well for 24 hours in DMEM+10\% FBS. Transfected cells were then trypsinized and used to seed 96-well plates at a density of $8.5 \mathrm{e} 4 \mathrm{cells} / \mathrm{well}$ and allowed to grow overnight. Dual luciferase assays were then performed using Promega Dual-Luciferase Reporter Assay System (Promega Cat\#E1910) following manufacturer protocol. Briefly, cells were lysed in $80 \mathrm{uL} 1 \mathrm{x}$ Passive Lysis buffer at room temperature for 15 minutes with vigorous agitation. After agitation, $50 \mathrm{uL}$ of lysate were transferred to a 96-well black bottom plate and read using dual injector luminometer set to dispense $50 \mathrm{uL}$ of reconstituted Luciferase assay substrate followed by Stop \& Glo buffer. For values, renilla luciferase (has the $3^{\prime} U T R$ insert) is normalized to firefly luciferase, which is a built-in control. Expression was than calculated by normalizing those plasmids with insert to empty control plasmid.

\section{Live cell imaging}

6-well glass bottom tissue culture plates (MatTek Corporation P06G-1.5-10-F.S.) were coated with 34 $\mathrm{ug} / \mathrm{mL}$ collagen (Sigma-Aldrich, C5533-5MG) for 1 hour at $37^{\circ} \mathrm{C}$ then seeded with cells grown in standard media until $70 \%$ confluence. Cells were treated with 100 uM sodium arsenite for 90 minutes in an onstage incubator $\left(37^{\circ} \mathrm{C}, 5 \% \mathrm{CO}_{2}\right)$ in conjunction with the Keyence BZ-X700 Microscope.

\section{Single molecule fluorescent in situ hybridization (smFISH)}

Single molecule FISH studies were done as described previously (Shaffer et al., 2013). Briefly, cells were grown on coverslips in 6-well plates until confluent. Cells were then fixed with $4 \%$ paraformaldehyde for 10 minutes followed by washing and permeabilization in $70 \%$ ethanol overnight at $4 \mathrm{C}$. Cells washed in FISH wash buffer (2x SSC, 10\% Formamide) then incubated overnight with probes in hybridization buffer $(10 \%$ dextran sulfate, $10 \%$ formamide, $2 \times$ SSC) at $37 \mathrm{C}$ at dilution of 1:100. After washing, cells stained with DAPI followed by wash in 2x SSC buffer before being mounted using VECTASHIELD Vibrance Antifade Mounting Medium (Vector Laboratories $\mathrm{H}-1700-2)$. Cells imaged using inverted Nikon Ti2-E microscope equipped with a SOLA SE U-nIR light engine (Lumencor), ORCA-Flash 4.0 V3 sCMOS camera (Hamamatsu), with $\times 60$ Plan-Apo $\lambda$ (MRD01605) or $\times 20$ Plan-Apo $\lambda$ (Nikon MRD00205). Specific probes are listed in Table 3. Images analyses were performed using ImageJ and CellProfiler (Dunn et al., 2011; McQuin et al., 2018).

\section{Statistical methods}

Data were analysed using unpaired, two-tailed student's t-tests, one-way ANOVA, or two-way ANOVA with post-hoc tests (Dunnett's or Tukey's) and p-values indicated in individual figures. Analyses were performed on data from a minimum of three experiments unless otherwise noted, in which case multiple fields are analysed. All values are presented as mean \pm standard error of mean (SEM) unless noted otherwise. Specific experimental replicates are described in each figure legend.

\section{References}

Abildgaard, M.H., S.H. Brynjolfsdottir, and L.B. Frankel. 2020. The Autophagy-RNA Interplay: Degradation and Beyond. Trends Biochem Sci. 45:845-857.

Adolph, T.E., M.F. Tomczak, L. Niederreiter, H.J. Ko, J. Bock, E. Martinez-Naves, J.N. Glickman, M. Tschurtschenthaler, J. Hartwig, S. Hosomi, M.B. Flak, J.L. Cusick, K. Kohno, T. Iwawaki, S. Billmann-Born, T. Raine, R. Bharti, R. Lucius, M.N. Kweon, S.J. Marciniak, A. Choi, S.J. Hagen, S. 
Schreiber, P. Rosenstiel, A. Kaser, and R.S. Blumberg. 2013. Paneth cells as a site of origin for intestinal inflammation. Nature. 503:272-276.

Asano, J., T. Sato, S. Ichinose, M. Kajita, N. Onai, S. Shimizu, and T. Ohteki. 2017. Intrinsic Autophagy Is Required for the Maintenance of Intestinal Stem Cells and for Irradiation-Induced Intestinal Regeneration. Cell Rep. 20:1050-1060.

Bel, S., M. Pendse, Y. Wang, Y. Li, K.A. Ruhn, B. Hassell, T. Leal, S.E. Winter, R.J. Xavier, and L.V. Hooper. 2017. Paneth cells secrete lysozyme via secretory autophagy during bacterial infection of the intestine. Science. 357:1047-1052.

Bell, J.L., K. Wachter, B. Muhleck, N. Pazaitis, M. Kohn, M. Lederer, and S. Huttelmaier. 2013. Insulin-like growth factor 2 mRNA-binding proteins (IGF2BPs): post-transcriptional drivers of cancer progression? Cell Mol Life Sci. 70:2657-2675.

Bley, N., M. Lederer, B. Pfalz, C. Reinke, T. Fuchs, M. Glass, B. Moller, and S. Huttelmaier. 2015. Stress granules are dispensable for mRNA stabilization during cellular stress. Nucleic Acids Res. 43:e26.

Bolt, A.M., R.M. Byrd, and W.T. Klimecki. 2010. Autophagy is the predominant process induced by arsenite in human lymphoblastoid cell lines. Toxicol Appl Pharmacol. 244:366-373.

Cadwell, K., J.Y. Liu, S.L. Brown, H. Miyoshi, J. Loh, J.K. Lennerz, C. Kishi, W. Kc, J.A. Carrero, S. Hunt, C.D. Stone, E.M. Brunt, R.J. Xavier, B.P. Sleckman, E. Li, N. Mizushima, T.S. Stappenbeck, and H.W.t. Virgin. 2008. A key role for autophagy and the autophagy gene Atg16l1 in mouse and human intestinal Paneth cells. Nature. 456:259-263.

Chatterji, P., K.E. Hamilton, S. Liang, S.F. Andres, H.R.S. Wijeratne, R. Mizuno, L.A. Simon, P.D. Hicks, S.W. Foley, J.R. Pitarresi, A.J. Klein-Szanto, A.T. Mah, L. Van Landeghem, B.D. Gregory, C.J. Lengner, B.B. Madison, P. Shah, and A.K. Rustgi. 2018. The LIN28B-IMP1 post-transcriptional regulon has opposing effects on oncogenic signaling in the intestine. Genes Dev. 32:1020-1034.

Chatterji, P., P.A. Williams, K.A. Whelan, F.C. Samper, S.F. Andres, L.A. Simon, L.R. Parham, R. Mizuno, E.T. Lundsmith, D.S. Lee, S. Liang, H.S. Wijeratne, S. Marti, L. Chau, V. Giroux, B.J. Wilkins, G.D. Wu, P. Shah, G.G. Tartaglia, and K.E. Hamilton. 2019. Posttranscriptional regulation of colonic epithelial repair by RNA binding protein IMP1/IGF2BP1. EMBO Rep. 20:e47074.

Conway, A.E., E.L. Van Nostrand, G.A. Pratt, S. Aigner, M.L. Wilbert, B. Sundararaman, P. Freese, N.J. Lambert, S. Sathe, T.Y. Liang, A. Essex, S. Landais, C.B. Burge, D.L. Jones, and G.W. Yeo. 2016. Enhanced CLIP Uncovers IMP Protein-RNA Targets in Human Pluripotent Stem Cells Important for Cell Adhesion and Survival. Cell Rep. 15:666-679.

Conway, K.L., P. Kuballa, J.H. Song, K.K. Patel, A.B. Castoreno, O.H. Yilmaz, H.B. Jijon, M. Zhang, L.N. Aldrich, E.J. Villablanca, J.M. Peloquin, G. Goel, I.A. Lee, E. Mizoguchi, H.N. Shi, A.K. Bhan, S.Y. Shaw, S.L. Schreiber, H.W. Virgin, A.F. Shamji, T.S. Stappenbeck, H.C. Reinecker, and R.J. Xavier. 2013. Atg16l1 is required for autophagy in intestinal epithelial cells and protection of mice from Salmonella infection. Gastroenterology. 145:1347-1357.

Copetti, T., C. Bertoli, E. Dalla, F. Demarchi, and C. Schneider. 2009. p65/RelA modulates BECN1 transcription and autophagy. Mol Cell Biol. 29:2594-2608.

Czarnomysy, R., D. Radomska, A. Muszynska, J.M. Hermanowicz, I. Prokop, A. Bielawska, and K. Bielawski. 2020. Evaluation of the Anticancer Activities of Novel Transition Metal Complexes with Berenil and Nitroimidazole. Molecules. 25:2860.

Dai, N., J. Christiansen, F.C. Nielsen, and J. Avruch. 2013. mTOR complex 2 phosphorylates IMP1 cotranslationally to promote IGF2 production and the proliferation of mouse embryonic fibroblasts. Genes Dev. 27:301-312.

Drake, K.R., M. Kang, and A.K. Kenworthy. 2010. Nucleocytoplasmic distribution and dynamics of the autophagosome marker EGFP-LC3. PLoS One. 5:e9806.

Dunn, K.W., M.M. Kamocka, and J.H. McDonald. 2011. A practical guide to evaluating colocalization in biological microscopy. Am J Physiol Cell Physiol. 300:C723-742.

Feng, Y., Z. Yao, and D.J. Klionsky. 2015. How to control self-digestion: transcriptional, post-transcriptional, and post-translational regulation of autophagy. Trends in cell biology. 25:354-363.

Frankel, L.B., M. Lubas, and A.H. Lund. 2017. Emerging connections between RNA and autophagy. Autophagy. 13:3-23.

Gorbunov, N.V., and J.G. Kiang. 2009. Up-regulation of autophagy in small intestine Paneth cells in response to total-body gamma-irradiation. The Journal of pathology. 219:242-252.

Guzikowski, A.R., Y.S. Chen, and B.M. Zid. 2019. Stress-induced mRNP granules: Form and function of processing bodies and stress granules. Wiley Interdiscip Rev RNA. 10:e1524. 
He, H., Y. Dang, F. Dai, Z. Guo, J. Wu, X. She, Y. Pei, Y. Chen, W. Ling, C. Wu, S. Zhao, J.O. Liu, and L. Yu. 2003. Post-translational modifications of three members of the human MAP1LC3 family and detection of a novel type of modification for MAP1LC3B. J Biol Chem. 278:29278-29287.

Huang, R., Y. Xu, W. Wan, X. Shou, J. Qian, Z. You, B. Liu, C. Chang, T. Zhou, J. Lippincott-Schwartz, and W. Liu. 2015. Deacetylation of nuclear LC3 drives autophagy initiation under starvation. Mol Cell. 57:456-466.

Hubstenberger, A., M. Courel, M. Benard, S. Souquere, M. Ernoult-Lange, R. Chouaib, Z. Yi, J.B. Morlot, A. Munier, M. Fradet, M. Daunesse, E. Bertrand, G. Pierron, J. Mozziconacci, M. Kress, and D. Weil. 2017. P-Body Purification Reveals the Condensation of Repressed mRNA Regulons. Mol Cell. 68:144-157 e145.

Huttelmaier, S., D. Zenklusen, M. Lederer, J. Dictenberg, M. Lorenz, X. Meng, G.J. Bassell, J. Condeelis, and R.H. Singer. 2005. Spatial regulation of beta-actin translation by Src-dependent phosphorylation of ZBP1. Nature. 438:512-515.

Ichimura, Y., T. Kirisako, T. Takao, Y. Satomi, Y. Shimonishi, N. Ishihara, N. Mizushima, I. Tanida, E. Kominami, M. Ohsumi, T. Noda, and Y. Ohsumi. 2000. A ubiquitin-like system mediates protein lipidation. Nature. 408:488-492.

Jing, Z., W. Han, X. Sui, J. Xie, and H. Pan. 2015. Interaction of autophagy with microRNAs and their potential therapeutic implications in human cancers. Cancer letters. 356:332-338.

Jonson, L., J. Vikesaa, A. Krogh, L.K. Nielsen, T. Hansen, R. Borup, A.H. Johnsen, J. Christiansen, and F.C. Nielsen. 2007. Molecular composition of IMP1 ribonucleoprotein granules. Mol Cell Proteomics. 6:798-811.

Klionsky, D.J., A.K. Abdel-Aziz, S. Abdelfatah, M. Abdellatif, A. Abdoli, S. Abel, H. Abeliovich, M.H. Abildgaard, Y.P. Abudu, A. Acevedo-Arozena, I.E. Adamopoulos, K. Adeli, T.E. Adolph, A. Adornetto, E. Aflaki, G. Agam, A. Agarwal, B.B. Aggarwal, M. Agnello, P. Agostinis, J.N. Agrewala, A. Agrotis, P.V. Aguilar, S.T. Ahmad, Z.M. Ahmed, U. Ahumada-Castro, S. Aits, S. Aizawa, Y. Akkoc, T. Akoumianaki, H.A. Akpinar, A.M. Al-Abd, L. Al-Akra, A. Al-Gharaibeh, M.A. Alaoui-Jamali, S. Alberti, E. Alcocer-Gomez, C. Alessandri, M. Ali, M.A. Alim Al-Bari, S. Aliwaini, J. Alizadeh, E. Almacellas, A. Almasan, A. Alonso, G.D. Alonso, N. Altan-Bonnet, D.C. Altieri, E.M.C. Alvarez, S. Alves, C. Alves da Costa, M.M. Alzaharna, M. Amadio, C. Amantini, C. Amaral, S. Ambrosio, A.O. Amer, V. Ammanathan, Z. An, S.U. Andersen, S.A. Andrabi, M. Andrade-Silva, A.M. Andres, S. Angelini, D. Ann, U.C. Anozie, M.Y. Ansari, P. Antas, A. Antebi, Z. Anton, T. Anwar, L. Apetoh, N. Apostolova, T. Araki, Y. Araki, K. Arasaki, W.L. Araujo, J. Araya, C. Arden, M.A. Arevalo, S. Arguelles, E. Arias, J. Arikkath, H. Arimoto, A.R. Ariosa, D. Armstrong-James, L. Arnaune-Pelloquin, A. Aroca, D.S. Arroyo, I. Arsov, R. Artero, D.M.L. Asaro, M. Aschner, M. Ashrafizadeh, O. AshurFabian, A.G. Atanasov, A.K. Au, P. Auberger, H.W. Auner, L. Aurelian, et al. 2021. Guidelines for the use and interpretation of assays for monitoring autophagy (4th edition)(1). Autophagy. 17:1-382.

Klionsky, D.J., Z. Elazar, P.O. Seglen, and D.C. Rubinsztein. 2008. Does bafilomycin A1 block the fusion of autophagosomes with lysosomes? Autophagy. 4:849-850.

Lambrianidou, A., E. Sereti, K. Soupsana, C. Komini, K. Dimas, and T. Trangas. 2021. mTORC2 deploys the mRNA binding protein IGF2BP1 to regulate c-MYC expression and promote cell survival. Cell Signal. 80:109912.

Lassen, K.G., P. Kuballa, K.L. Conway, K.K. Patel, C.E. Becker, J.M. Peloquin, E.J. Villablanca, J.M. Norman, T.C. Liu, R.J. Heath, M.L. Becker, L. Fagbami, H. Horn, J. Mercer, O.H. Yilmaz, J.D. Jaffe, A.F. Shamji, A.K. Bhan, S.A. Carr, M.J. Daly, H.W. Virgin, S.L. Schreiber, T.S. Stappenbeck, and R.J. Xavier. 2014. Atg16L1 T300A variant decreases selective autophagy resulting in altered cytokine signaling and decreased antibacterial defense. Proc Natl Acad Sci U S A. 111:7741-7746.

Lipinski, M.M., G. Hoffman, A. Ng, W. Zhou, B.F. Py, E. Hsu, X. Liu, J. Eisenberg, J. Liu, J. Blenis, R.J. Xavier, and J. Yuan. 2010. A genome-wide siRNA screen reveals multiple mTORC1 independent signaling pathways regulating autophagy under normal nutritional conditions. Developmental cell. 18:1041-1052.

Liu, M., G. Tao, Y. Cao, Y. Hu, and Z. Zhang. 2022. Silencing of IGF2BP1 restrains ox-LDL-induced lipid accumulation and inflammation by reducing RUNX1 expression and promoting autophagy in macrophages. J Biochem Mol Toxicol:e22994.

Mateu-Regue, A., J. Christiansen, F.O. Bagger, O. Winther, C. Hellriegel, and F.C. Nielsen. 2019. Single mRNP Analysis Reveals that Small Cytoplasmic mRNP Granules Represent mRNA Singletons. Cell Rep. 29:736-748 e734. 
Mauthe, M., I. Orhon, C. Rocchi, X. Zhou, M. Luhr, K.J. Hijlkema, R.P. Coppes, N. Engedal, M. Mari, and F. Reggiori. 2018. Chloroquine inhibits autophagic flux by decreasing autophagosome-lysosome fusion. Autophagy. 14:1435-1455.

Mauvezin, C., and T.P. Neufeld. 2015. Bafilomycin A1 disrupts autophagic flux by inhibiting both VATPase-dependent acidification and Ca-P60A/SERCA-dependent autophagosome-lysosome fusion. Autophagy. 11:1437-1438.

McQuin, C., A. Goodman, V. Chernyshev, L. Kamentsky, B.A. Cimini, K.W. Karhohs, M. Doan, L. Ding, S.M. Rafelski, D. Thirstrup, W. Wiegraebe, S. Singh, T. Becker, J.C. Caicedo, and A.E. Carpenter. 2018. CellProfiler 3.0: Next-generation image processing for biology. PLoS Biol. 16:e2005970.

Mikhaylova, O., Y. Stratton, D. Hall, E. Kellner, B. Ehmer, A.F. Drew, C.A. Gallo, D.R. Plas, J. Biesiada, J. Meller, and M.F. Czyzyk-Krzeska. 2012. VHL-regulated MiR-204 suppresses tumor growth through inhibition of LC3B-mediated autophagy in renal clear cell carcinoma. Cancer Cell. 21:532-546.

Mizushima, N., and T. Yoshimori. 2007. How to interpret LC3 immunoblotting. Autophagy. 3:542-545.

Moor, A.E., M. Golan, E.E. Massasa, D. Lemze, T. Weizman, R. Shenhav, S. Baydatch, O. Mizrahi, R. Winkler, O. Golani, N. Stern-Ginossar, and S. Itzkovitz. 2017. Global mRNA polarization regulates translation efficiency in the intestinal epithelium. Science. 357:1299-1303.

Nakatogawa, H., Y. Ichimura, and Y. Ohsumi. 2007. Atg8, a ubiquitin-like protein required for autophagosome formation, mediates membrane tethering and hemifusion. Cell. 130:165-178.

Niewidok, B., M. Igaev, A. Pereira da Graca, A. Strassner, C. Lenzen, C.P. Richter, J. Piehler, R. Kurre, and R. Brandt. 2018. Single-molecule imaging reveals dynamic biphasic partition of RNA-binding proteins in stress granules. J Cell Biol. 217:1303-1318.

Nighot, P.K., C.A. Hu, and T.Y. Ma. 2015. Autophagy enhances intestinal epithelial tight junction barrier function by targeting claudin-2 protein degradation. J Biol Chem. 290:7234-7246.

Rangan, P., I. Choi, M. Wei, G. Navarrete, E. Guen, S. Brandhorst, N. Enyati, G. Pasia, D. Maesincee, V. Ocon, M. Abdulridha, and V.D. Longo. 2019. Fasting-Mimicking Diet Modulates Microbiota and Promotes Intestinal Regeneration to Reduce Inflammatory Bowel Disease Pathology. Cell Rep. 26:2704-2719 e2706.

Rioux, J.D., R.J. Xavier, K.D. Taylor, M.S. Silverberg, P. Goyette, A. Huett, T. Green, P. Kuballa, M.M. Barmada, L.W. Datta, Y.Y. Shugart, A.M. Griffiths, S.R. Targan, A.F. Ippoliti, E.J. Bernard, L. Mei, D.L. Nicolae, M. Regueiro, L.P. Schumm, A.H. Steinhart, J.I. Rotter, R.H. Duerr, J.H. Cho, M.J. Daly, and S.R. Brant. 2007. Genome-wide association study identifies new susceptibility loci for Crohn disease and implicates autophagy in disease pathogenesis. Nature genetics. 39:596-604.

Rodriguez, A.E., C. Lopez-Crisosto, D. Pena-Oyarzun, D. Salas, V. Parra, C. Quiroga, T. Morawe, M. Chiong, C. Behl, and S. Lavandero. 2016. BAG3 regulates total MAP1LC3B protein levels through a translational but not transcriptional mechanism. Autophagy. 12:287-296.

Sadaghian Sadabad, M., A. Regeling, M.C. de Goffau, T. Blokzijl, R.K. Weersma, J. Penders, K.N. Faber, H.J. Harmsen, and G. Dijkstra. 2015. The ATG16L1-T300A allele impairs clearance of pathosymbionts in the inflamed ileal mucosa of Crohn's disease patients. Gut. 64:1546-1552.

Shaffer, S.M., M.T. Wu, M.J. Levesque, and A. Raj. 2013. Turbo FISH: a method for rapid single molecule RNA FISH. PLoS One. 8:e75120.

Stohr, N., M. Lederer, C. Reinke, S. Meyer, M. Hatzfeld, R.H. Singer, and S. Huttelmaier. 2006. ZBP1 regulates mRNA stability during cellular stress. J Cell Biol. 175:527-534.

Thoreen, C.C., S.A. Kang, J.W. Chang, Q. Liu, J. Zhang, Y. Gao, L.J. Reichling, T. Sim, D.M. Sabatini, and N.S. Gray. 2009. An ATP-competitive mammalian target of rapamycin inhibitor reveals rapamycinresistant functions of mTORC1. J Biol Chem. 284:8023-8032.

Trentesaux, C., M. Fraudeau, C.L. Pitasi, J. Lemarchand, S. Jacques, A. Duche, F. Letourneur, E. Naser, K. Bailly, A. Schmitt, C. Perret, and B. Romagnolo. 2020. Essential role for autophagy protein ATG7 in the maintenance of intestinal stem cell integrity. Proc Natl Acad Sci U S A. 117:11136-11146.

Wachter, K., M. Kohn, N. Stohr, and S. Huttelmaier. 2013. Subcellular localization and RNP formation of IGF2BPs (IGF2 mRNA-binding proteins) is modulated by distinct RNA-binding domains. Biol Chem. 394:1077-1090.

Yamamoto, A., Y. Tagawa, T. Yoshimori, Y. Moriyama, R. Masaki, and Y. Tashiro. 1998. Bafilomycin A1 prevents maturation of autophagic vacuoles by inhibiting fusion between autophagosomes and lysosomes in rat hepatoma cell line, H-4-II-E cells. Cell Struct Funct. 23:33-42.

Yee, K.S., S. Wilkinson, J. James, K.M. Ryan, and K.H. Vousden. 2009. PUMA- and Bax-induced autophagy contributes to apoptosis. Cell death and differentiation. 16:1135-1145. 
Yoshii, S.R., and N. Mizushima. 2017. Monitoring and Measuring Autophagy. Int J Mol Sci. 18:1865.

Yousefi, M., A. Nakauka-Ddamba, C.T. Berry, N. Li, J. Schoenberger, K.P. Simeonov, R.J. Cedeno, Z. Yu, and C.J. Lengner. 2018. Calorie Restriction Governs Intestinal Epithelial Regeneration through CellAutonomous Regulation of mTORC1 in Reserve Stem Cells. Stem Cell Reports. 10:703-711.

Zeng, W.J., C. Lu, Y. Shi, C. Wu, X. Chen, C. Li, and J. Yao. 2020. Initiation of stress granule assembly by rapid clustering of IGF2BP proteins upon osmotic shock. Biochim Biophys Acta Mol Cell Res. 1867:118795.

\section{Figure Legends}

Figure 1: IMP1 knockout is associated with increased expression of MAP1LC3 protein. A. Western blot of whole cell lysates from IMP1-WT or IMP1-KO SW480 cells treated with 20nM torin or $50 \mathrm{~nm}$ bafilomycin A1 (Baf) for 3 hours and probed with antibody specific to MAP1LC3B. For MAP1LC3B blots, two concentrations of lysates are presented to facilitate accurate quantification of LC3-II (10ug) and to visualize both LC3-I and LC3-II (20ug), respectively. B. Densitometry analysis of western blots represented in Figure 1A. LC3-II quantification normalized to GAPDH. Data are expressed as mean $\pm \mathrm{SEM}, \mathrm{N}=4 .{ }^{* * *} \mathrm{p}=$ $0.0002 ;{ }^{* * *} p<0.0001$. C. Western blot of lysates from IMP1-WT or IMP1-KO SW480 cells treated with bafilomycin $\mathrm{A} 1$ at indicated concentrations for 24 hours and probed with antibody that recognizes both MAP1LC3A and MAP1LC3B. D. Densitometry analysis of western blot in Figure 1C. LC3-II quantification normalized to GAPD. Data are presented as mean \pm SEM. F. Representative immunofluorescent images of IMP1-WT and IMP1-KO SW480 cells stained with antibody for MAP1LC3A/B. Note increased MAP1LC3A/B in IMP1-KO compared to IMP1-WT cells. Images representative of $\mathrm{n}=3$ fields of view for $\mathrm{n}=$ 2 independent experiments.

Figure 2: IMP1 phosphorylation (p-IMP1) contributes to regulation of MAP1LC3B expression. A. Schematic of IMP1 phosphorylation sites at serine-181 (S-181) and tyrosine-396 (Y-396) B. Western blot of whole cell lysates from HEK-293 IMP1-KO cells with stable expression of indicated GFP constructs: control-GFP (IMP1-KO), IMP1-WT-GFP, IMP1-S181A-GFP, IMP1-Y369F-GFP, IMP1-S181A/Y396F-GFP. Blots probed with IMP1, MAP1LC3B, and GAPDH antibodies. C. Densitometry analysis of western blots represented in Figure 2B. MAP1LC3B normalized to GAPDH. Data are expressed as mean \pm SEM. $N=3$. ${ }^{* *} p=0.006$. E. Representative flow cytometry plots of Autophagy Assay, Red fluorescence intensity in control (red) and chloroquine treated (3 hours, 100 $\mu \mathrm{m}$; blue) control-GFP (IMP1-KO), IMP1-WT-GFP, IMP1-S181A-GFP, IMP1-Y369F-GFP, and IMP1-S181A/Y396F-GFP cells normalized to mode. E. Quantification of fold change in median fluorescence intensity (MFI) with chloroquine treatment between all five cell lines listed in $D$. Data are expressed as mean \pm SEM. $N \geq 3$. ${ }^{*} p=0.03$.

\section{Figure 3: IMP1 knockout is associated with increased MAP1LC3B-3'UTR translation A.}

Immunofluorescence of HEK-293 IMP1-KO cells with stable expression of indicated GFP constructs shown with IMP1-GFP (Green) and DAPI (blue) shown. Sample cell nuclei are indicated with white dotted lines. B. Schematic of dual-luciferase reporter assay with MAP1LC3B-3'UTR cloned into siCHECK2 plasmid.

Plasmids were transiently transfected at equal concentrations into control-GFP (IMP1-KO), IMP1-WT-GFP, and IMP1-S181A/Y396F-GFP cells. B. Dual luciferase reporter assays were performed to compare relative luciferase activity of MAP1LC3B-3'UTR compared to empty control in each of the cell lines described in B. Renilla luciferase was normalized to built-in control firefly luciferase. Data are presented as mean \pm SEM. $N$ $\geq 3 .{ }^{*} p=0.03$.

\section{Figure 4: IMP1 partially co-localizes with stress granule marker PABP in response to cell stress.} Immunofluorescence of HEK-293 IMP1-KO cells with stable expression of IMP1-WT-GFP or IMP1S181A/Y396F-GFP and treated with $100 \mathrm{uM}$ of sodium arsenite or vehicle control (water) for 45 minutes A. Staining for GFP (green), EDC4 (red), and DAPI (blue) in IMP1-WT-GFP-expressing cells. White arrows in insets of vehicle control denote examples of GFP-EDC4 co-localization that is lost with arsenite treatment. B. GFP (green), EDC4 (red), and DAPI (blue) in S181A/Y396F-GFP-expressing cells. White arrows in vehicle control denote GFP-EDC4 co-localization that is decreased with arsenite treatment. Note EDC4 granules appear reduced in arsenite treated IMP1- S181A/Y396F-GFP compared to IMP1-WT-GFP cells. C. Staining for GFP (green), PABP (red), and DAPI (blue). White arrows in arsenite-treated insets denote overlap of GFP and PABP in IMP1-WT-GFP-expressing cells. D. GFP (green), PABP (red), and 
DAPI (blue) staining in S181A/Y396F-GFP-expressing cells. Note an overall decrease in granule formation, but that GFP and PABP+ are still apparent (white arrows in insets). $\mathrm{N} \geq 2$ independent experiments and images are representative of 3 fields of view per experiment.

Figure 5: IMP1-GFP colocalization with MAP1LC3B transcript is reduced following acute cell stress. A. Immunofluorescence of HEK-293 IMP1-KO cells transfected with IMP1-WT-GFP (green) and MAP1LC3B mRNA (red) using probes listed in Table 3. Cells were treated with $100 \mathrm{uM}$ sodium arsenite or water control for 45 minutes. Nuclei are stained with DAPI (blue). White arrows in vehicle-treated cells indicate IMP1-MAP1LC3B mRNA co-localization. B. Immunofluorescence in IMP1-WT-GFP cells with IMP1-WT-GFP (green), ATG5 mRNA (red). Note lack of GFP- ATG5 mRNA co-localization. C. Immunofluorescence of HEK-293 IMP1-KO cells transfected with IMP1-S181A/Y396F-GFP (green) and MAP1LC3B mRNA (red). Note a reduction in IMP1-MAP1LC3B mRNA co-localization compared to A (vehicle-treated). D. Immunofluorescence of cells described in C with IMP1-S181A/Y396F-GFP (green), ATG5 mRNA (red). E. Correlation analysis of images in 5A \& C (vehicle) using CellProfiler to quantify overlap of MAP1LC3 mRNA and IMP1 fluorescence. Rank weight coefficient analysis was performed on $\mathrm{n}$ $\geq 5$ high-powered fields across two independent experiments. Data expressed as SEM. ${ }^{* *} p=0.01 . F$. Distribution of MAP1LC3B within indicated cell lines from perinuclear (1) to cell periphery (4). $N \geq 4$ images analysed across two independent experiments using CellProfiler with mean fraction distribution.

\section{Figure 6: Model of IMP1 regulation of MAP1LC3B}

Our data support a new mechanism for post-transcriptional regulation of MAP1LC3B by IMP1. IMP1 sequestration of MAP1LC3B transcripts in cytoplasmic granules may poise homeostatic cells to respond to stress by protecting MAP1LC3B mRNA in potentially diverse ribonucleoprotein (RNP) granules, including processing bodies. Upon stress, or with mutation of IMP1 phosphorylation sites (p-IMP1), IMP1MAP1LC3B mRNA co-localization is reduced, where IMP1 is localized to stress granules and MAP1LC3B transcripts are observed further from the nucleus. Our observation that MAP1LC3B protein is increased with IMP1 deletion supports a model in which reduction of IMP1-MAP1LC3B mRNA co-localization permits increased translation of MAP1LC3B. Schematic created with BioRender (Biorender.com).

Supplemental Figure 1: A. Western blot of SW480 whole cell lysates from IMP1-WT or IMP1-KO cells treated with either $20 \mathrm{nM}$ Torin1 or DMSO (control) for 24 hours and probed with antibody that recognizes both MAP1LC3A and MAP1LC3B, as well as other antibodies listed. B. Densitometry analysis of western blots represented in Supplemental Figure 1A. LC3-II was normalized to GAPDH. Data are expressed as mean \pm SEM, $N=3$. ${ }^{*} p=.04$. C. Real-time RT-PCR on total RNA from SW480 IMP1-WT or IMP1-KO cells treated with either $20 \mathrm{nM}$ torin1 or vehicle (DMSO) for 24 hours. Gene expression for MAP1LC3B was normalized to GAPDH expression. Data are expressed as mean \pm SEM versus IMP1-WT. $\mathrm{N}=3$.

Supplemental Figure 2: Schematic of Autophagy Probe, Red assay in Figure 2D, E.

Supplemental Figure 3: A. Western blot of lysates from fractionated HEK-293 IMP1-KO cells with stable expression of IMP1-WT-GFP or IMP1-S181A-GFP using the Subcellular Protein Fractionation Kit for Cultured Cells. Blots are probed with indicated antibodies to indicate nuclear and cytosolic fractions. Cytosolic Extraction (CEB), Membrane Extraction (MEB), Nuclear Extraction (NEB), Chromatin Bound Extraction (NEB-C), Cytoskeletal Extraction (PEB) lysates are represented. B. Densitometry of western blots indicate significantly more cytosolic than nuclear IMP1 in IMP1-WT-GFP, but not IMP1-S181A-GFP cells. Cytosolic included CEB and MEB fractions, nuclear included NEB and NEB-C fractions. Data are presented as mean \pm SEM with $p$-values indicated. $\mathrm{N}=3$.

Movie S1. Time-lapse imaging of arsenite-treated IMP1-WT-GFP cells.

IMP1-WT-GFP cells exhibited robust cytoplasmic granule formation with arsenite treatment. Cells were treated with $100 \mathrm{uM}$ sodium arsenite for 90 minutes. https://doi.org/10.6084/m9.figshare.16304799.

\section{Movie S2. Time-lapse imaging of arsenite-treated IMP1-S181A-GFP cells.}

IMP1-S181A-GFP cells exhibited reduced overall granule formation after arsenite treatment compared to IMP1-WT-GFP cells. Cells were treated with 100 uM sodium arsenite for 90 minutes.

https://doi.org/10.6084/m9.figshare.16304802. 
Movie S3. Time-lapse imaging of arsenite-treated IMP1-Y-396F-GFP cells.

IMP1-Y-396F-GFP cells exhibited reduced overall granule formation after arsenite treatment compared to IMP1-WT-GFP cells. Cells were treated with 100 uM sodium arsenite for 90 minutes.

https://doi.org/10.6084/m9.figshare.17162717.v1.

Table 1: Antibodies

\begin{tabular}{|l|l|l|l|}
\hline Antibody & Manufacturer & Catalogue Number & Dilution (application, block) \\
\hline IMP1 & Cell Signaling & D33A2 & $1: 1000$ (western blotting, milk) \\
\hline MAP1LC3A/B & Cell Signaling & 12741 & $\begin{array}{l}1: 1000 \text { (western blotting, milk), } \\
1: 100 \text { (immunofluorescence) }\end{array}$ \\
\hline MAP1LC3B (D11) & Cell Signaling & 3868 & $1: 1000$ (western blotting, milk) \\
\hline SQSTM1/p62 & Abcam & Ab56416 & $1: 1000$ (western blotting, milk) \\
\hline ATG5 & Cell Signaling & $12994 S$ & $1: 1000$ (western blotting, milk) \\
\hline ATG3 & Cell Signaling & $3415 T$ & $1: 1000$ (western blotting, milk) \\
\hline pS6RP (Ser235/236) & Cell Signaling & $4858 S$ & $1: 1000$ (western blotting, BSA) \\
\hline S6RP & Cell Signaling & $2217 S$ & $1: 1000$ (western blotting, milk) \\
\hline GAPDH & Santa Cruz & sc-32233 & $1: 2000$ (western blotting, milk) \\
\hline HDAC2 & Cell Signaling & $2540 S$ & $1: 1000$ (western blotting, milk) \\
\hline EDC4 & Santa Cruz & Sc-374211 & $1: 100$ (immunofluorescence) \\
\hline GFP & Novus Bio & NB100-1614 & $1: 200$ (immunofluorescence) \\
\hline PABP & Santa Cruz & sc-32318 & $1: 100$ (immunofluorescence) \\
\hline
\end{tabular}

Table 2: qPCR assays

\begin{tabular}{|l|l|}
\hline Gene target & Assay number \\
\hline GAPDH & Hs02758991_g1 \\
\hline MAP1LC3B & Hs01076567_g1 \\
\hline
\end{tabular}

Table 3 smFISH Probes

\begin{tabular}{|l|l|}
\hline agctgcatttttctggttt & MAP1LC3B_1 \\
\hline actgatcgatctcagttggt & MAP1LC3B_2 \\
\hline actactgtttcatgatctgt & MAP1LC3B_3 \\
\hline gctcagttttctgcttgaa & MAP1LC3B_4 \\
\hline ccaaagctgaatgtgctcac & MAP1LC3B_5 \\
\hline gtctctctgaaagcctttaa & MAP1LC3B_6 \\
\hline acttctacccaaaaaggcgg & MAP1LC3B_7 \\
\hline & \\
gatctctctagctgagtaga & MAP1LC3B_8 \\
\hline & \\
cttcacaactcaggcctaaa & MAP1LC3B_9 \\
\hline
\end{tabular}




\begin{tabular}{|c|c|}
\hline aaatgcgttttgcgggggtt & MAP1LC3B_10 \\
\hline cgtttgtgccaactgtgatg & MAP1LC3B_11 \\
\hline tttacagtcagggccgtttt & MAP1LC3B_12 \\
\hline taggagtcagggaccttcag & MAP1LC3B_13 \\
\hline tgaggactttgggtgtggtt & MAP1LC3B_14 \\
\hline gaaatgtctacccctgcaag & MAP1LC3B_15 \\
\hline tttctccagggtcttttaag & MAP1LC3B 16 \\
\hline ggcactagtttgaactcaga & MAP1LC3B_17 \\
\hline tgctgctttccgtaacaaca & MAP1LC3B_18 \\
\hline tgtactccagagtgacactg & MAP1LC3B_19 \\
\hline tactatttgtgtttctccc & MAP1LC3B_20 \\
\hline agggagtgtgtctgaatgtt & MAP1LC3B_21 \\
\hline tggatcatccacagctttaa & MAP1LC3B_22 \\
\hline gtgggtaccttttcagtaat & MAP1LC3B_23 \\
\hline ctgtctgacctaagagtgat & MAP1LC3B_24 \\
\hline tggtgtgggagaattctgat & MAP1LC3B_25 \\
\hline ttctccacaactgacatgca & MAP1LC3B_26 \\
\hline gtacggctcttttgctatg & MAP1LC3B_27 \\
\hline ctcttgacattagtatctgt & MAP1LC3B_28 \\
\hline atcacaggttgaacctgagg & MAP1LC3B_29 \\
\hline
\end{tabular}




\begin{tabular}{|c|c|}
\hline tactgggaagcactagtctt & MAP1LC3B_30 \\
\hline taaatagtgaaccccatgca & MAP1LC3B_31 \\
\hline cacatagggtctaaagtggt & MAP1LC3B_32 \\
\hline acaagggtaagttgtgacct & MAP1LC3B_33 \\
\hline caggtatttcatacacatct & MAP1LC3B_34 \\
\hline ttccccgttacagtaaacag & MAP1LC3B_35 \\
\hline cccagatgcaaagaatctgg & MAP1LC3B_36 \\
\hline tgacaggaactcctttaaca & MAP1LC3B_37 \\
\hline tctaaacgagacagtccagt & MAP1LC3B_38 \\
\hline ctgatcacacggtagttaca & MAP1LC3B_39 \\
\hline gaccactcacatgggatata & MAP1LC3B_40 \\
\hline gacagttttaattgtgcct & MAP1LC3B_41 \\
\hline cagcgaatacaaccggcaac & ATG5_1 \\
\hline caaagccaaacctactccaa & ATG5_2 \\
\hline gtcattcttccaggagttaa & ATG5_3 \\
\hline tctcgaagcacatctttgtc & ATG5_4 \\
\hline tggaattcgtccaaaccaca & ATG5_5 \\
\hline ctttcagttatctcatcctg & ATG5_6 \\
\hline acttgtcagttaccaacgt & ATG5_7 \\
\hline tgtcttcttgtctcataacc & ATG5_8 \\
\hline ggataatgccatttcagtgg & ATG5_9 \\
\hline gtgatgttccaaggaagagc & ATG5_10 \\
\hline ttgcatccttagatggacag & ATG5_11 \\
\hline cagagttgcttgtgatcttt & ATG5_12 \\
\hline gcccaaaactggtcaaatct & ATG5_13 \\
\hline attccatgagtttccgattg & ATG5_14 \\
\hline aatccattttcttctgcagg & ATG5_15 \\
\hline ctttcagtcgttgtctgata & ATG5_16 \\
\hline ttctttgaggagatctccta & ATG5_17 \\
\hline
\end{tabular}




\begin{tabular}{|c|c|}
\hline agaggtgtttccaacattgg & ATG5_18 \\
\hline ggatgattctgttcaggcaa & ATG5_19 \\
\hline acatgctctgataaatccca & ATG5_20 \\
\hline aagtgtttctggtcaggttg & ATG5_21 \\
\hline gccttatctgacatggaatc & ATG5_22 \\
\hline gatgcaaagtaagaccagcc & ATG5_23 \\
\hline ggtttaatgatggcagtgga & ATG5_24 \\
\hline aagtctttcatgtcacagct & ATG5_25 \\
\hline atacccctgtttatttgtta & ATG5_26 \\
\hline aaagttcaaccatggtcacc & ATG5_27 \\
\hline ccaatgtttccactttcatg & ATG5_28 \\
\hline agtaatcctatgccctatat & ATG5_29 \\
\hline gcttggaaggaatgggtttt & ATG5_30 \\
\hline ttagacctggagccaatgaa & ATG5_31 \\
\hline ttcaaaagactggctgccag & ATG5_32 \\
\hline ctgcagtcaagagacaggta & ATG5_33 \\
\hline cccagaggcttggtaataaa & ATG5_34 \\
\hline gccttttagcgtactcaaat & ATG5_35 \\
\hline accatgatcgtgtctgatac & ATG5_36 \\
\hline tatctggtctttgaccaact & ATG5_37 \\
\hline agattgtatgctgtactggc & ATG5_38 \\
\hline tggcactttcgtttacatca & ATG5_39 \\
\hline gtcagtggattttttatcct & ATG5_40 \\
\hline caatcatattcagaccagcc & ATG5_41 \\
\hline ccccaaaatgaaccgacgaa & ATG5_42 \\
\hline ctgatccttttgctcactaa & ATG5_43 \\
\hline cttcatcacagcttagtgtt & ATG5_44 \\
\hline tgacactggtttgtctgta & ATG5_45 \\
\hline cactcaagcctactgcaaag & ATG5_46 \\
\hline gacttacctgggattagagt & ATG5_47 \\
\hline atttctggttttactcttcc & ATG5_48 \\
\hline
\end{tabular}


Figure 1

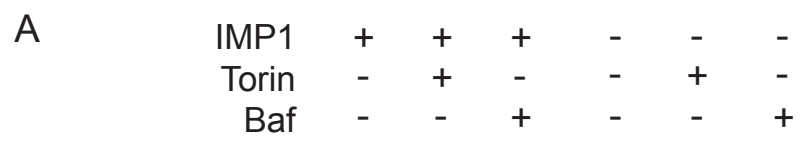

B LC3-II (10ug)

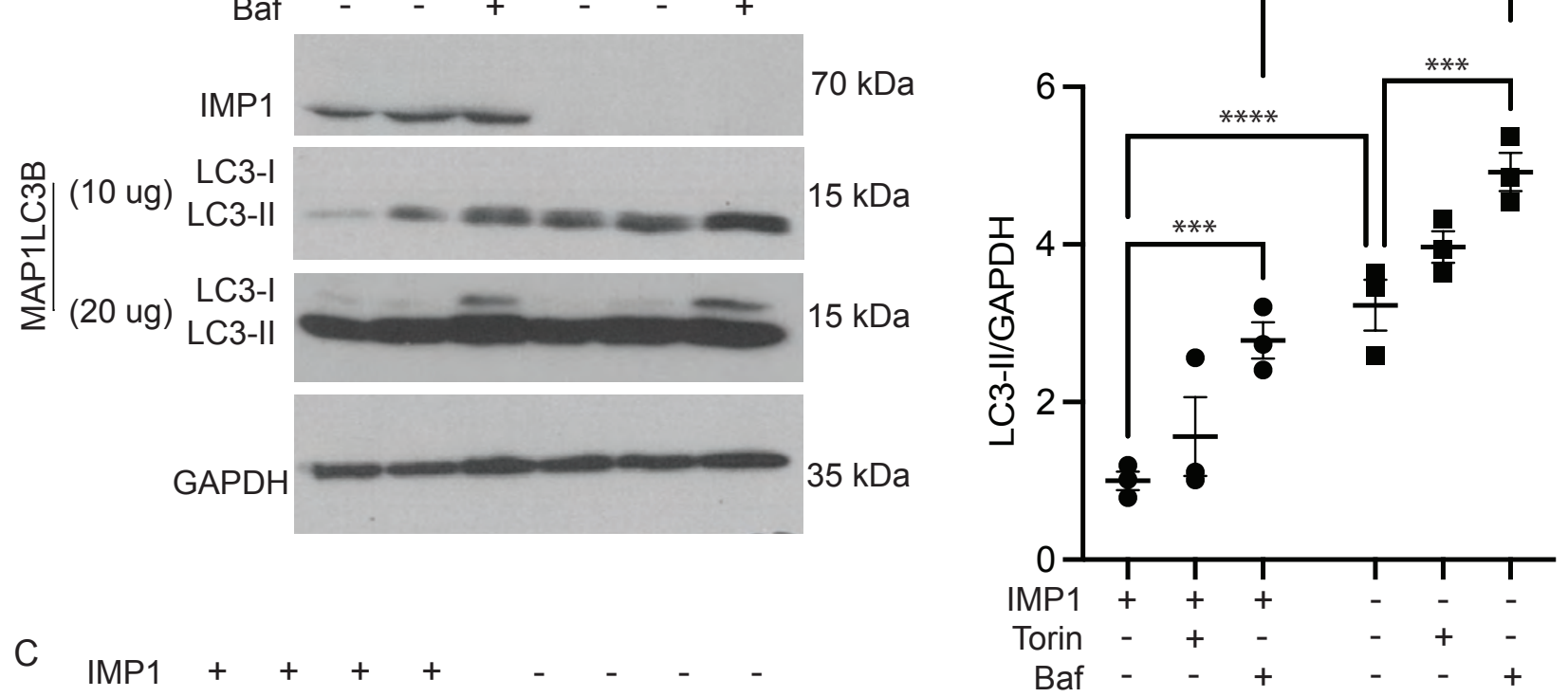

$\begin{array}{lllllllll}\mathrm{BafA}(\mathrm{nM}) & 0 & 50 & 100 & 200 & 0 & 50 & 100 & 200\end{array}$
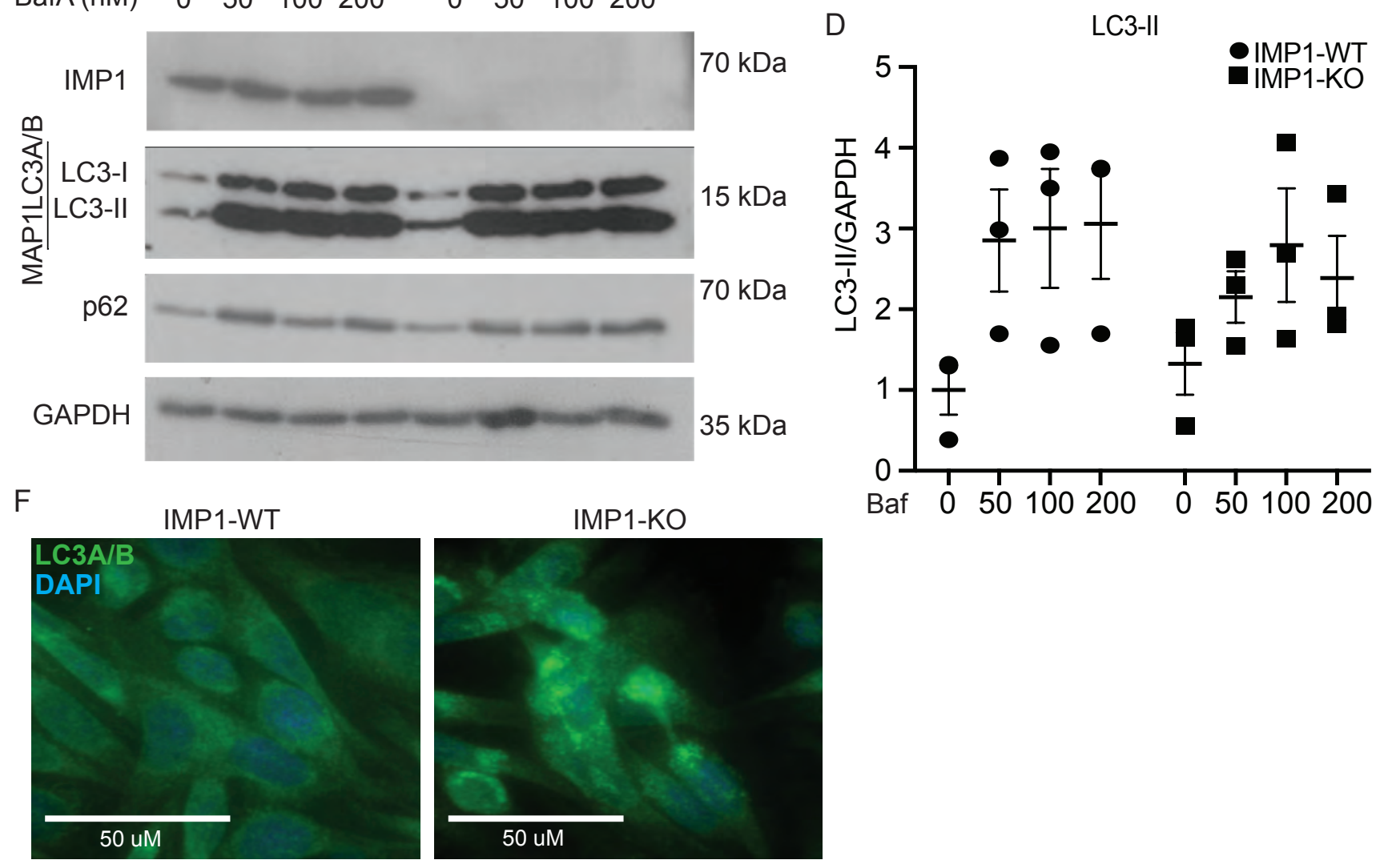
Figure 2

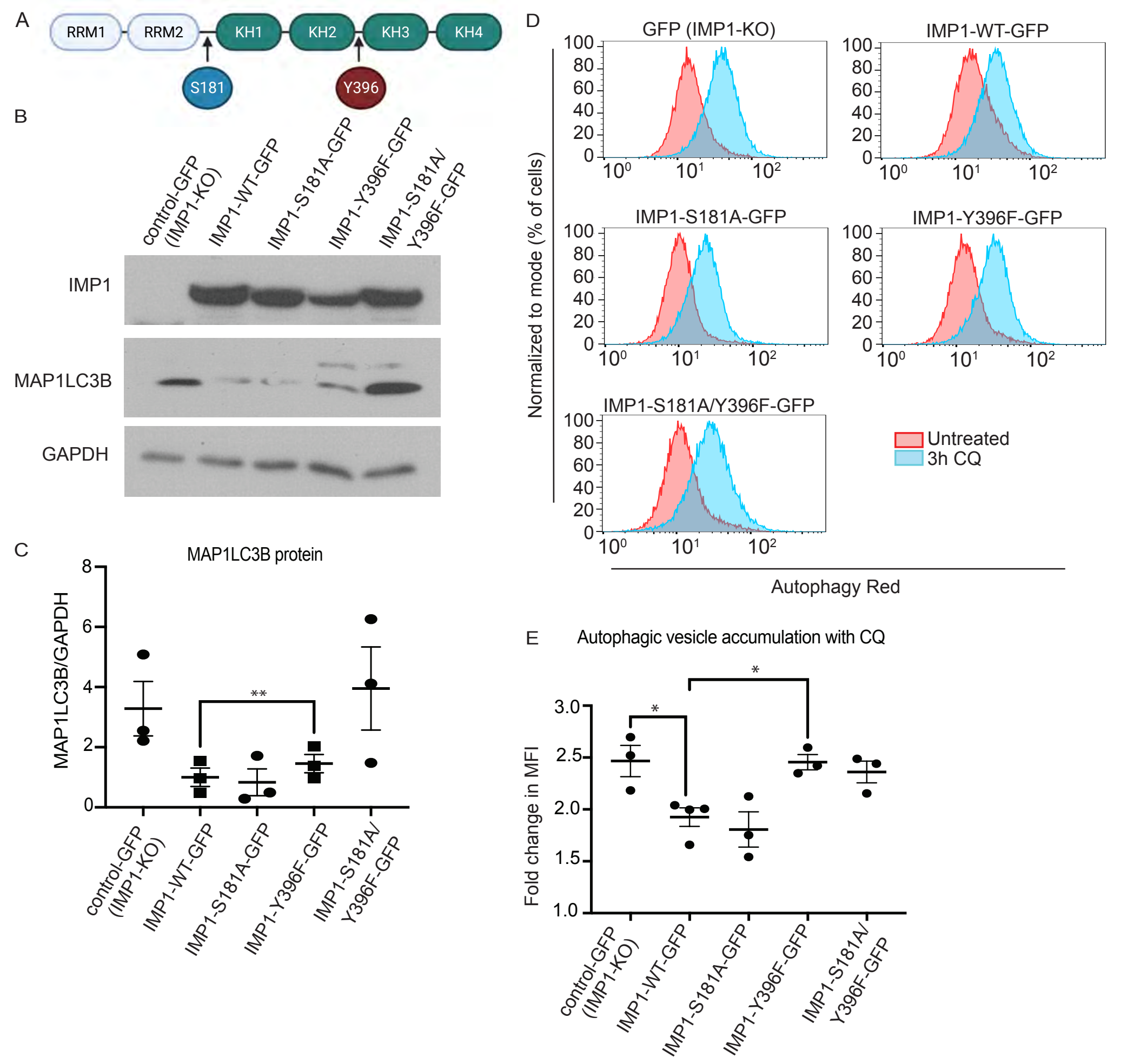


Figure 3
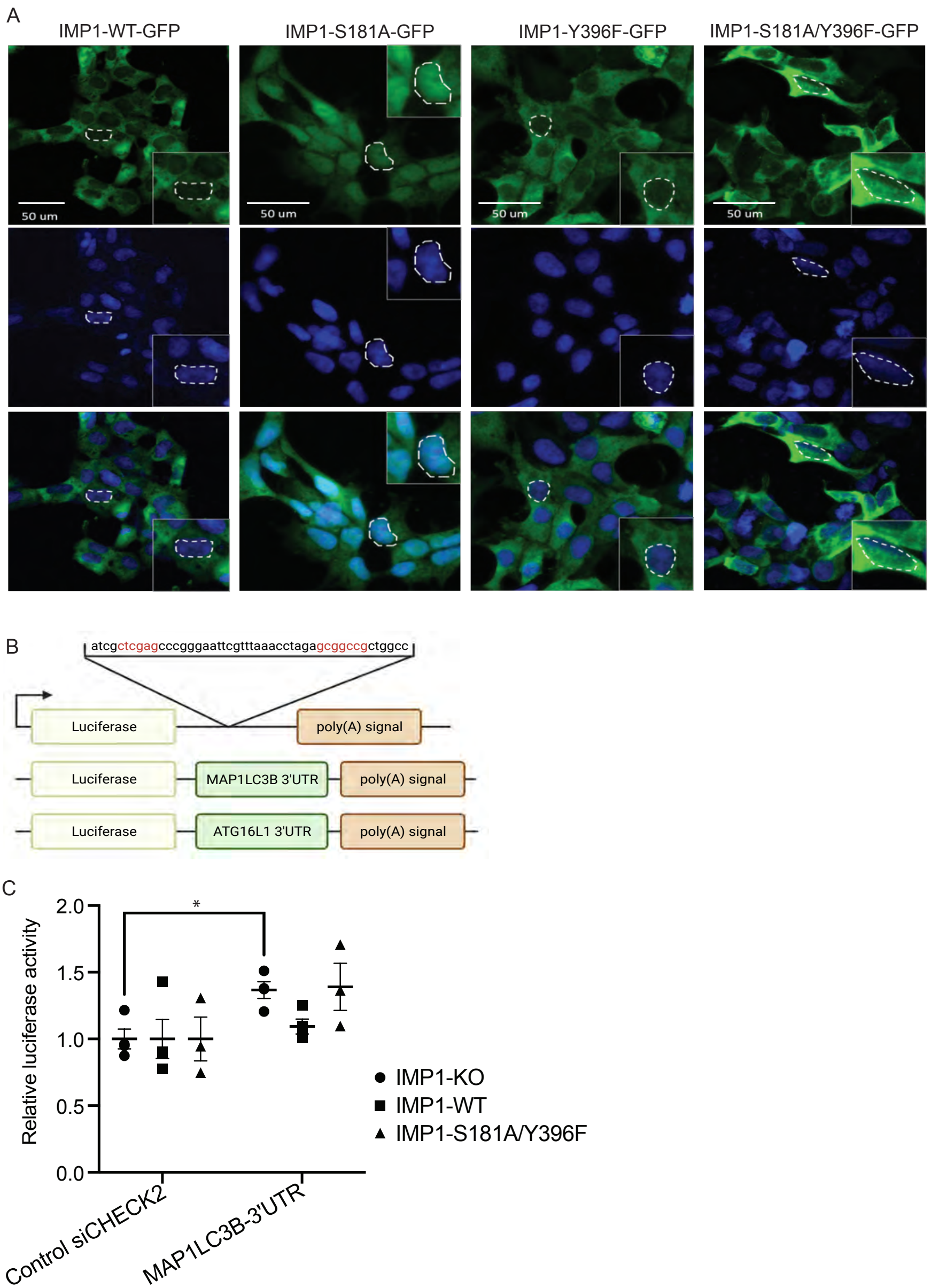
Figure 4

A

IMP1-WT-GFP
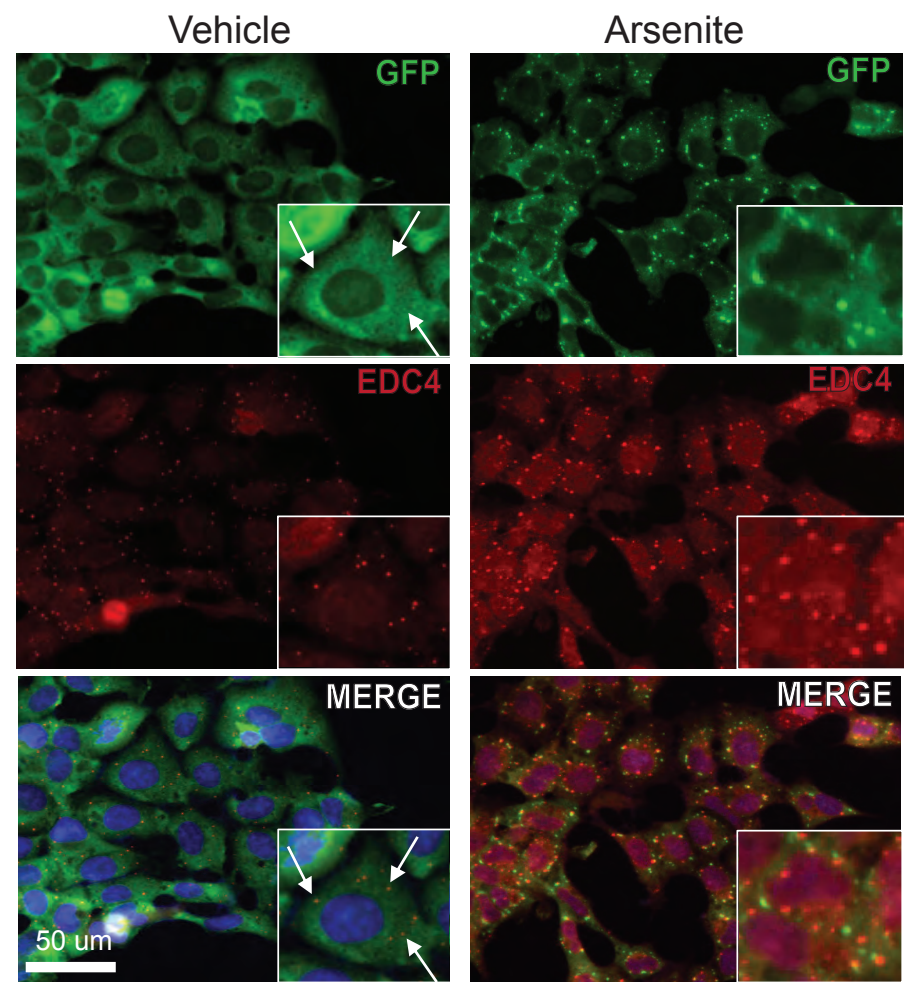

C

IMP1-WT-GFP

Vehicle
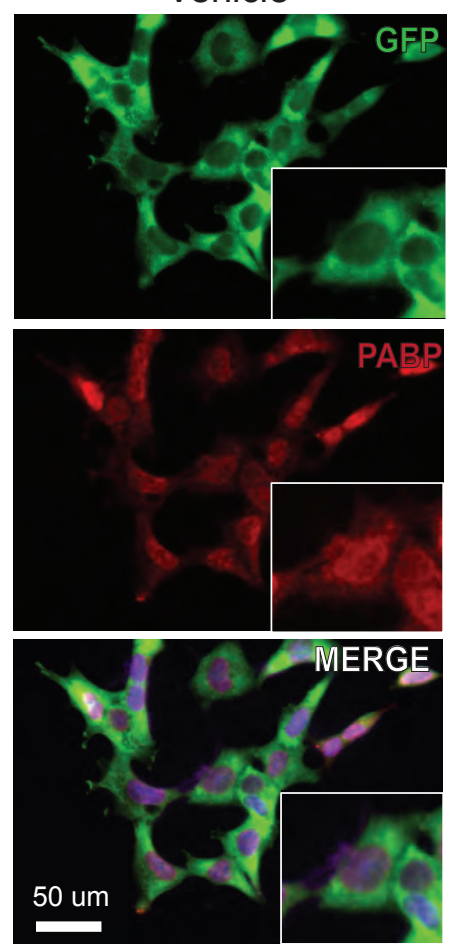

Arsenite
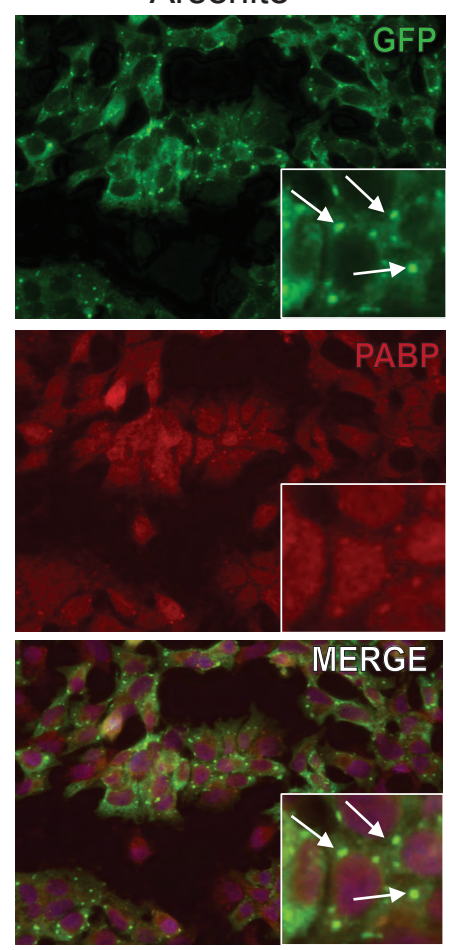

MERGE
B IMP1-S181/Y396F-GFP
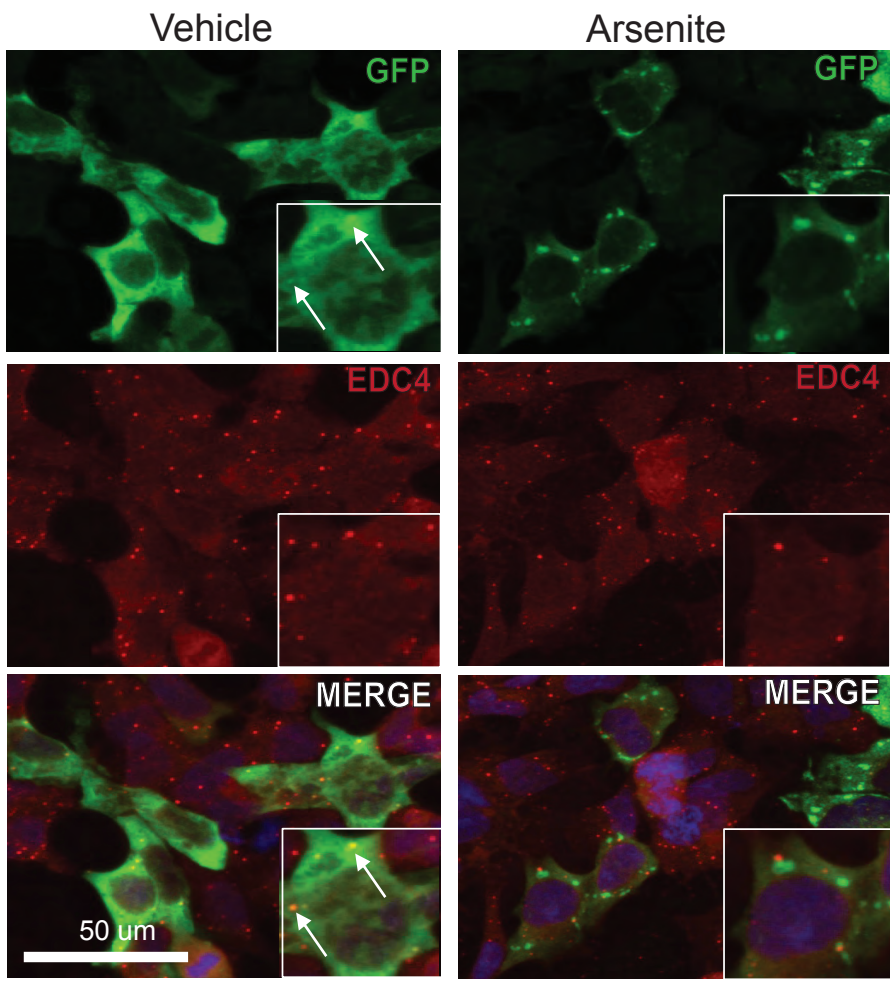

D

IMP1-S181/Y396F-GFP Vehicle

Arsenite
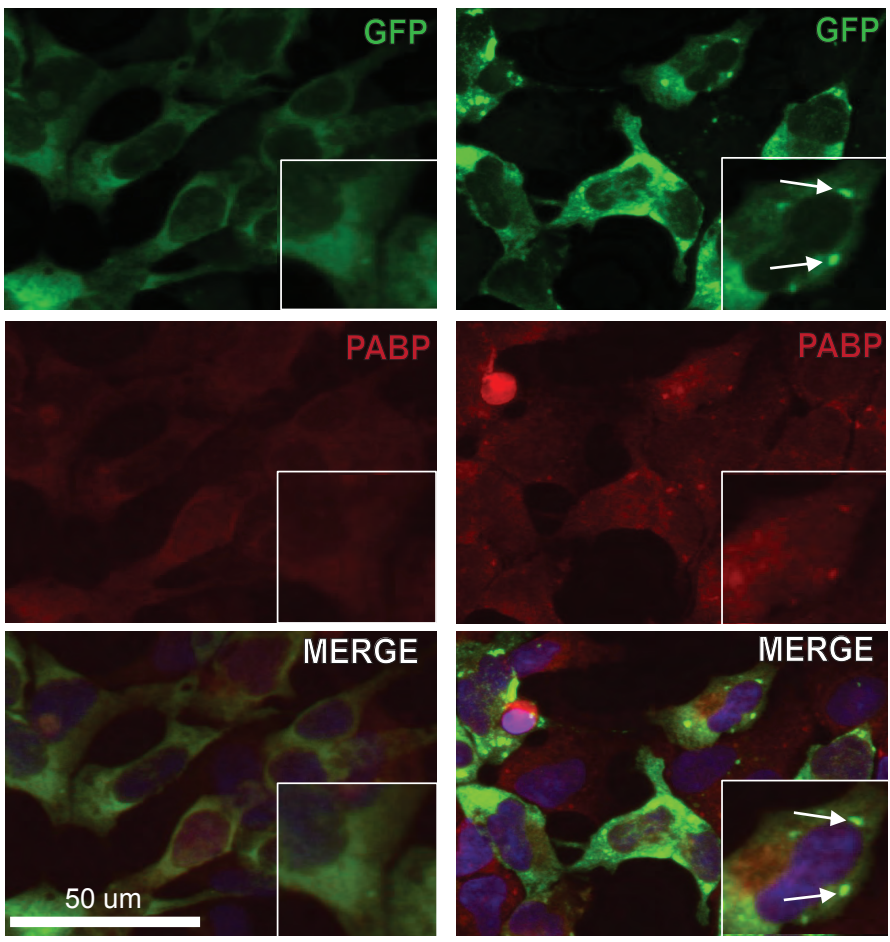
Figure 5

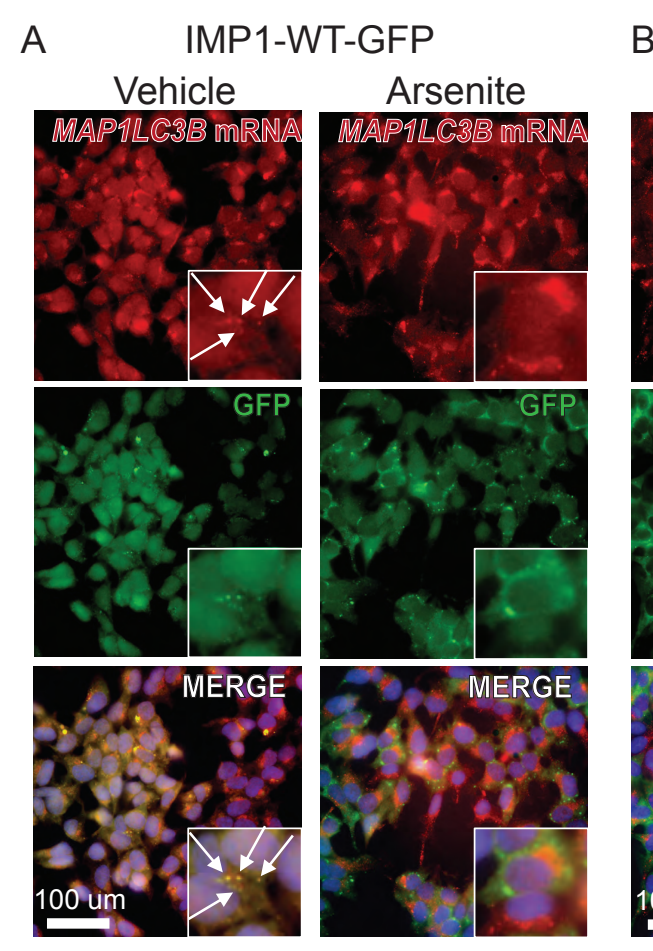

B IMP1-WT-GFP Vehicle Arsenite
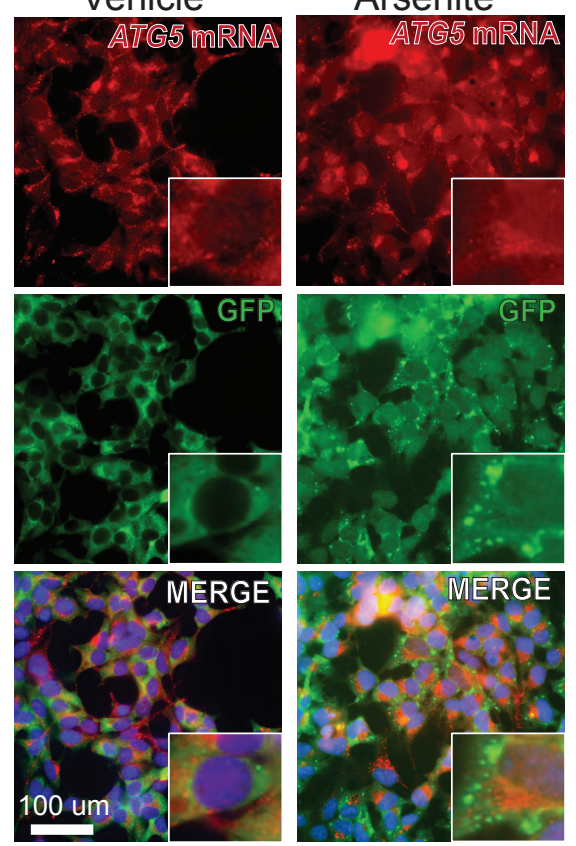

C IMP1-S181A/Y396F-GFP Vehicle
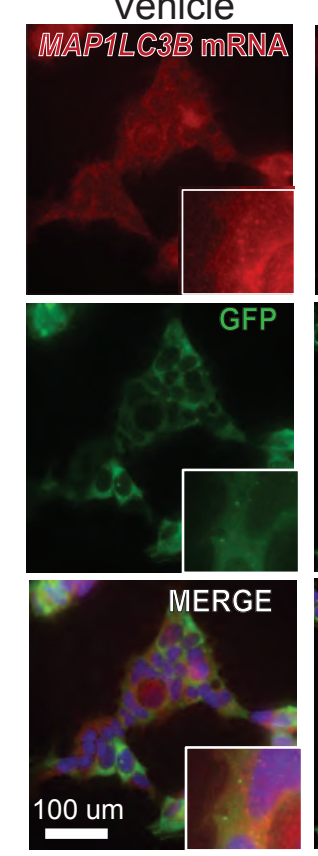

Arsenite
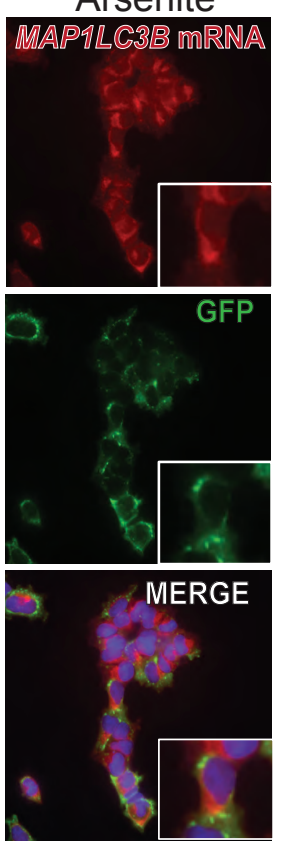

D IMP1-S181A/Y396F-GFP

Vehicle

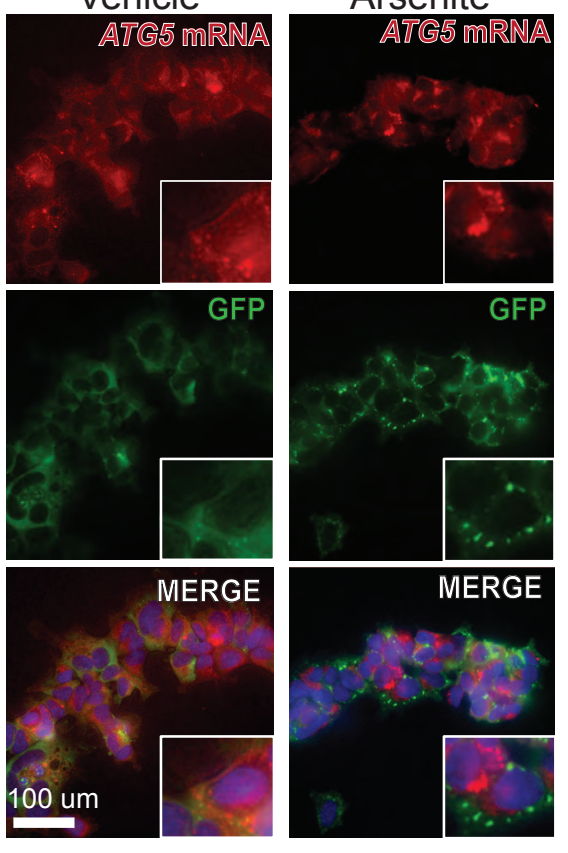

$\mathrm{E}$

Co-localization between IMP1-GFP and MAP1LC3B mRNA

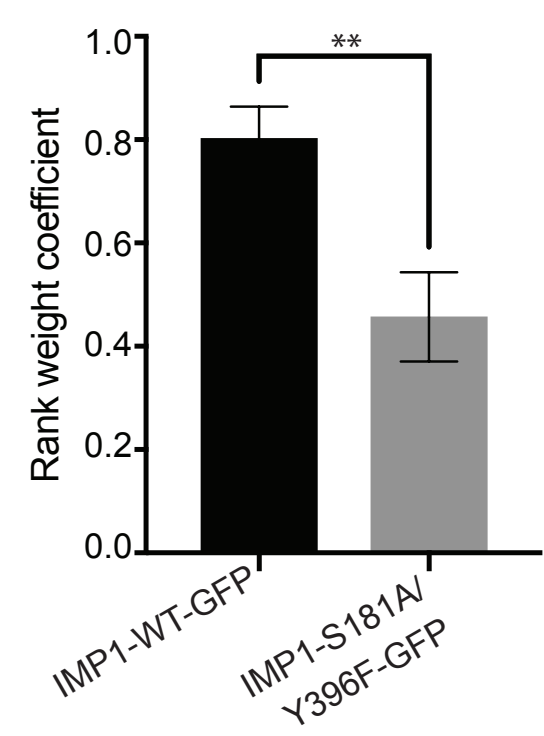

$\mathrm{F}$

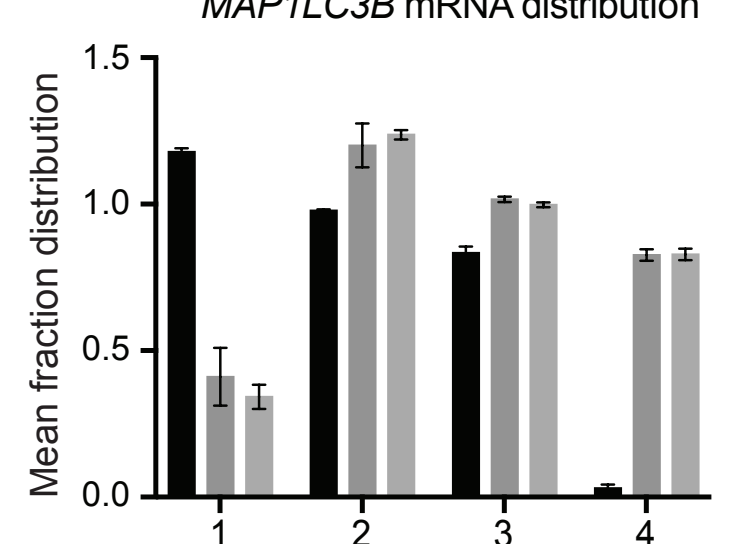

Distance from the nucleus (perinuclear to periphery)
IMP1-WT

IMP1-S181/Y396F

IMP1-WT + arsenite 
Figure 6

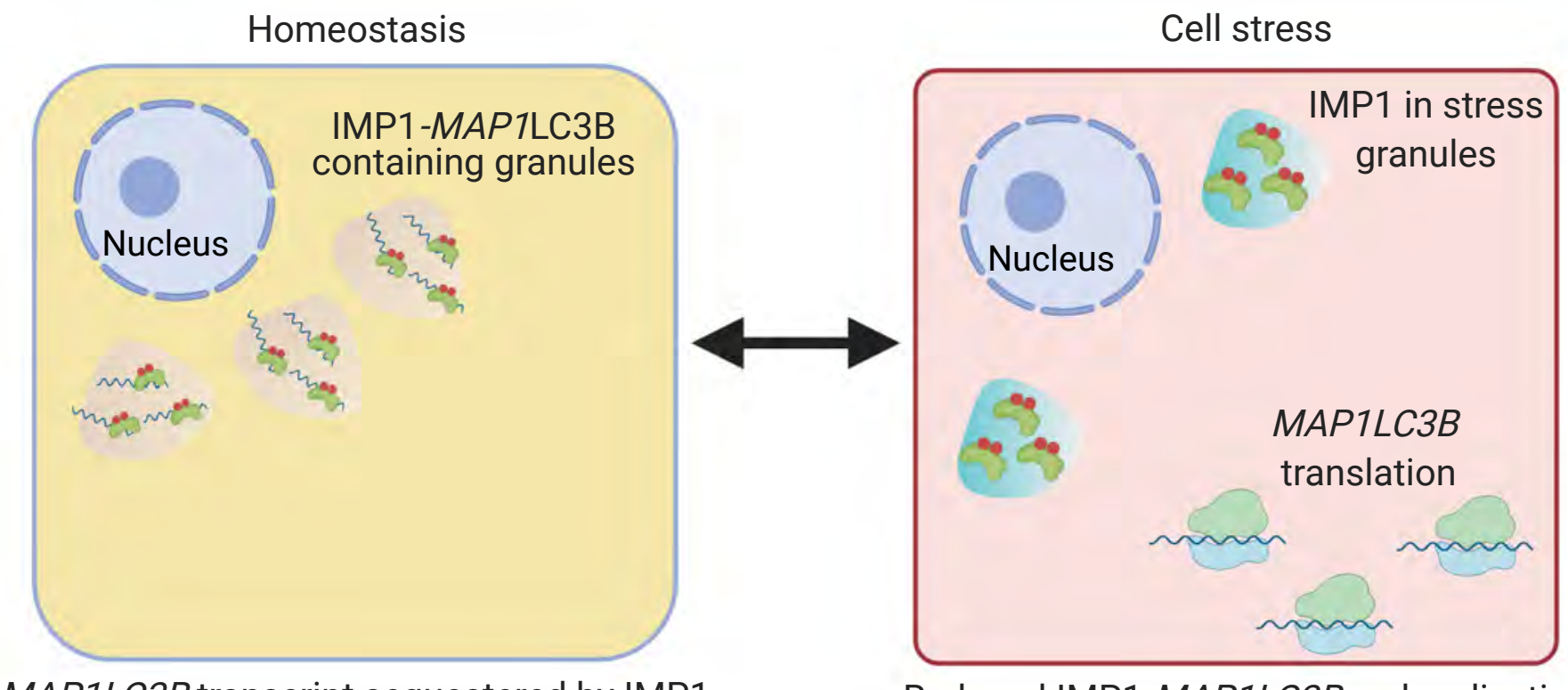

Schematic Key

m MAP1LC3B MRNA

p-IMP1

Processing body (or other RNP granules)

Ribosome

Stress granule 


\section{Supplemental Figure 1}
$\begin{array}{r}\text { A IMP1 } \\ \text { Torin }\end{array} \quad+\quad+\quad-\quad+$
B LC3-II protein
C MAP1LC3B mRNA
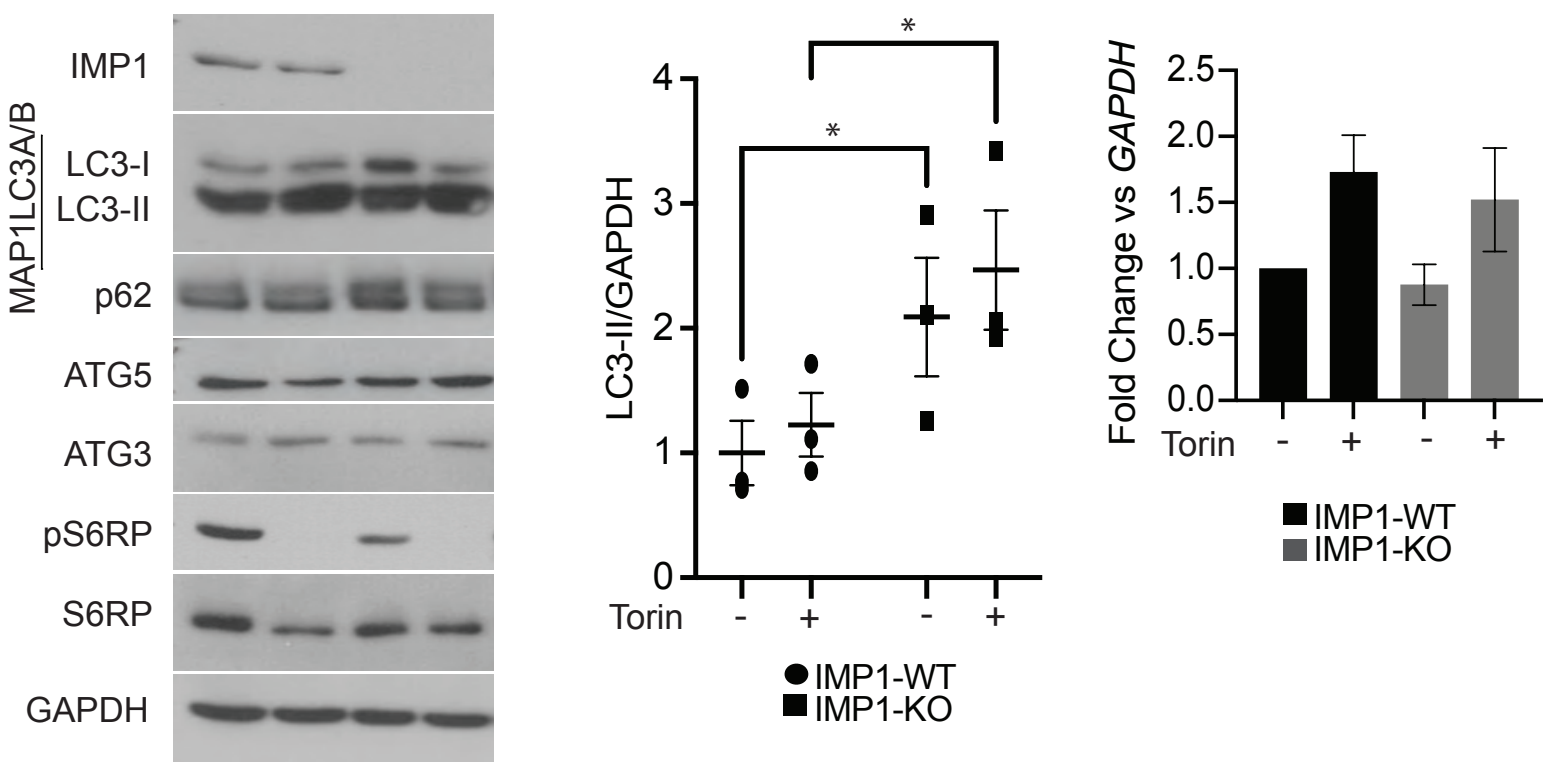


\section{Supplemental Figure 2}

A $+C Q-C Q$ (control)

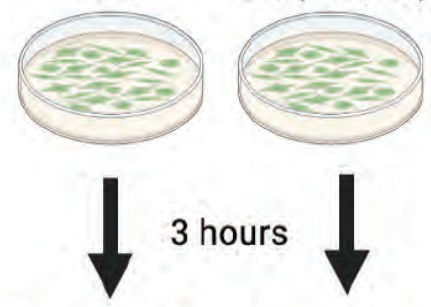

Autophagy Probe, Red stain $\left(30 \mathrm{~min}\right.$ at $\left.37^{\circ} \mathrm{C}\right)$
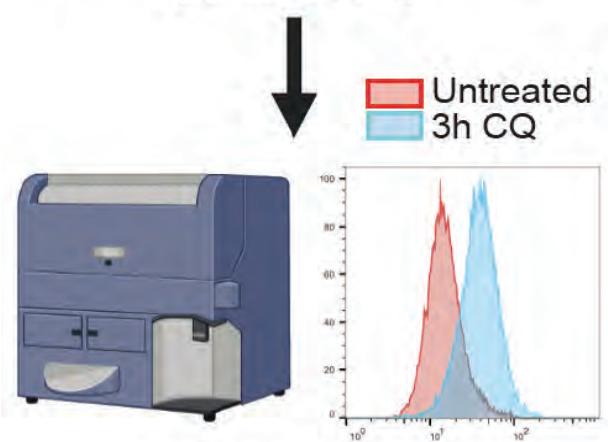

FACS analysis 


\section{Supplemental Figure 3}
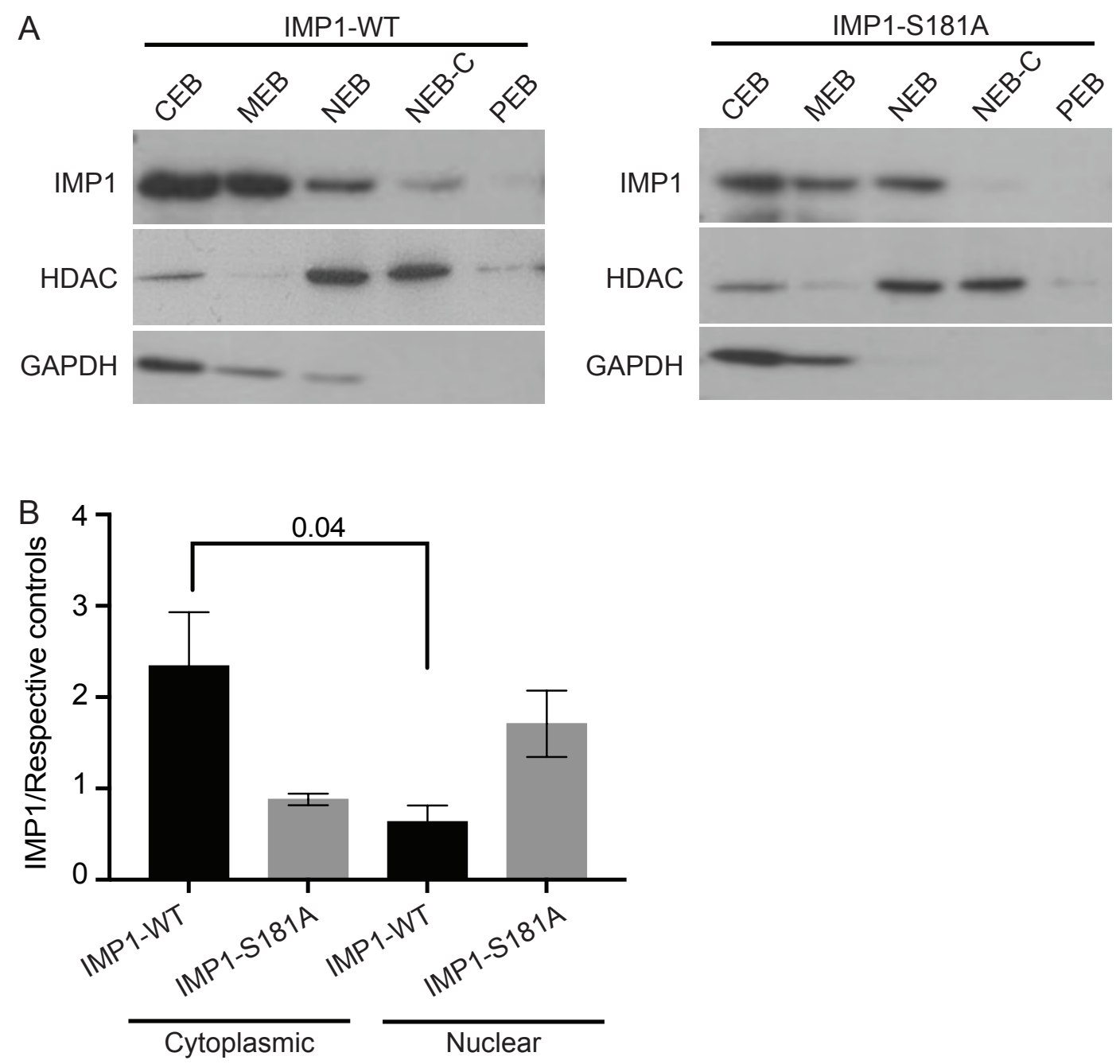